\title{
SOFIA/FORCAST Observations of the Sgr A-H H II Regions: Using Dust Emission to Elucidate the Heating Sources
}

\author{
M. J. Hankins ${ }^{1}$ (1D), R. M. Lau ${ }^{2}$, E. A. C. Mills ${ }^{3}$ (10), M. R. Morris ${ }^{4}$ (1) and T. L. Herter ${ }^{5}$ (10) \\ ${ }^{1}$ Division of Physics, Mathematics, and Astronomy, California Institute of Technology, Pasadena, CA 91125, USA; mhankins@caltech.edu \\ ${ }^{2}$ Institute of Space \& Astronautical Science, Japan Aerospace Exploration Agency, 3-1-1 Yoshinodai, Chuo-ku, Sagamihara, Kanagawa 252-5210, Japan \\ Physics Department, Brandeis University, 415 South Street, Waltham, MA 02453, USA \\ ${ }^{4}$ Department of Physics and Astronomy, University of California, Los Angeles, CA 90095-1547, USA \\ ${ }^{5}$ Department of Astronomy, Cornell University, Space Sciences Bldg., Ithaca, NY 14853-6801, USA \\ Received 2018 July 9; revised 2019 February 10; accepted 2019 March 15; published 2019 May 20
}

\begin{abstract}
The Sgr A-H H II regions are a collection of sources that may represent one of the most recent sites of star formation in the Galactic center (GC). Examining these $\mathrm{H}$ II regions provides important information on the prevalence and distribution of massive stars in the region and may provide clues on the origin of other massive field stars throughout the GC. In this work, we present infrared imaging observations of the Sgr A-H H II regions taken with the Faint Object Infrared Camera for the SOFIA Telescope (FORCAST) at 19.7, 25.2, 31.5, and $37.1 \mu \mathrm{m}$. These data provide high angular resolution maps $\left(\sim 4^{\prime \prime}\right)$ of the sources, which allow us to study the morphology of spatially extended H II regions in detail. The wavelength coverage of FORCAST also supplies important constraints on the dust mass, temperature, and luminosity of the sources. We produce spectral energy distribution models using DustEM to measure the luminosity and dust mass, which range from $\sim 3 \times 10^{3}$ to $6 \times 10^{5} L_{\odot}$ and from $\sim 6 \times 10^{-4}$ to $3 \times 10^{-1} M_{\odot}$, respectively. Analysis of dust temperatures in the four spatially extended $\mathrm{H}$ II regions ( $\mathrm{H} 1, \mathrm{H} 2, \mathrm{H} 3$, and $\mathrm{H} 5)$ shows that three of these objects require multiple heating sources, and we identify potential stellar candidates. We also compare the size and morphology of the H II regions and demonstrate that the properties of $\mathrm{H} 2$ are consistent with in situ star formation. Lastly, we identify nine additional sources that may be part of the $\mathrm{H}$ complex and provide initial characterizations of their infrared emission.
\end{abstract}

Key words: Galaxy: center - H II regions

\section{Introduction}

The spatial distribution of massive stars in the Galactic center (GC) is highly peculiar. Mauerhan et al. (2010) find that roughly half of the supergiant $\mathrm{O}$ and Wolf-Rayet stars present in the inner $\sim 90 \mathrm{pc}$ are isolated from known stellar clusters. $\mathrm{N}$ body simulations of cluster evolution in the GC environment by Habibi et al. (2014) suggest that many of these isolated stars could be former members of the Arches cluster (Cotera et al. 1996; Figer et al. 1999) or Quintuplet cluster (Nagata et al. 1990; Okuda et al. 1990) that have been tidally stripped or ejected. However, this may not be the origin of all of the field stars, as some fraction of them may have formed in situ. While it is widely accepted that stars generally form in clusters (McKee \& Ostriker 2007), our knowledge of clustered star formation in the GC is limited to only a few relatively large clusters $\left(L_{\text {cluster }} \gtrsim 10^{7} L_{\odot} ;\right.$ Figer 2004). It is reasonable to expect more modest-sized clusters to be present as well, though finding these sources has been elusive (e.g., Steinke et al. 2016). Examining the field stars in the region may reveal the location of new clusters and shed further insights on the process of star formation in this complex environment.

As a whole, star formation in the GC $\left(\mathrm{SFR}_{\mathrm{GC}} \approx 0.1 M_{\odot} \mathrm{yr}^{-1}\right.$; Barnes et al. 2017) is a factor of $\sim 10$ less efficient than one would expect based on its dense molecular gas content (Longmore et al. 2013). While star formation in the region is deficient, there there are still sites of ongoing and recent star formation, such as Sgr B2 (with more than 60 ultracompact and hypercompact H II regions; Goldsmith et al. 1990; Gaume et al. 1995; de Pree et al. 1996; De Pree et al. 1998) and the Sgr A East $\mathrm{H}$ II regions (with three compact and one ultracompact H II region; Ekers et al. 1983; Yusef-Zadeh et al. 2010; Mills et al.
2011; Lau et al. 2014). Both of these regions are believed to be examples of in situ star formation and comprise very different scales of star formation activity. Detailed examination of these regions and similar sites may help us better understand the inefficient nature of star formation in the GC, which is thought to be a result of turbulent interstellar matter (ISM) conditions that are primarily driven by large-scale instabilities (Kruijssen et al. 2014).

In this work, we have examined a complex of a 13 H II regions, known as the Sgr A-H H II regions (henceforth the $\mathrm{H}$ $\mathrm{H}$ II regions following the naming convention of Yusef-Zadeh $\&$ Morris 1987). These sources are located $\sim 10-15 \mathrm{pc}$ in projection from $\mathrm{Sgr}^{*}$ and were first discovered in radio mapping of Sgr A by Yusef-Zadeh \& Morris (1987). The H II regions are associated with the -30 to $0 \mathrm{~km} \mathrm{~s}^{-1}$ molecular cloud (Serabyn \& Guesten 1987; Poglitsch et al. 1991), which is hypothesized to lie on the far side of the GC (Molinari et al. 2011; Kruijssen et al. 2014), although the origin of their associated stars is debated. The Hubble Space Telescope (HST) $\mathrm{Pa} \alpha$ survey of the GC (Wang et al. 2010) identified a handful of emission-line stars in this region, and subsequent studies of these sources have revealed a few that are likely associated with and responsible for ionizing the H II regions (e.g., Dong et al. 2015, 2017). However, the majority of the H II regions still do not have an identified candidate ionization source.

Dynamical simulations by Habibi et al. (2014) demonstrate that stripped or evaporated stars from the Arches cluster could be found in this location, indicating that the $\mathrm{H}$ II regions may be formed by escaped, massive stars fortuitously passing through a molecular cloud. However, there is evidence that at least one of the sources $(\mathrm{H} 2)$ is associated with a star that likely formed 
in situ (Dong et al. 2015). Because the origin of many of these sources is uncertain, it is possible that additional $\mathrm{H}$ II regions in the complex have also formed in this manner. Examining the thermal dust emission can be an effective way to differentiate these scenarios, especially if the emission is resolved. However, dust emission associated with these sources is badly saturated at $24 \mu \mathrm{m}$ in the Spitzer/MIPS observations of the GC (e.g., Hinz et al. 2009), and earlier observations with the Midcourse Space Experiment (Egan et al. 2003) lack sufficient angular resolution to cleanly separate many of the infrared sources in this region.

To improve our understanding of the $\mathrm{H} \mathrm{H}$ II regions and their origin, we have obtained imaging observations of these sources at 19.7, 25.2, 31.5, and 37.1 $\mu \mathrm{m}$ using the Faint Object Infrared Camera for the SOFIA Telescope (FORCAST; Herter et al. 2012). In this paper, we present an analysis of these observations that trace thermal emission from warm dust $(\sim 100 \mathrm{~K})$ associated with the H II regions. Details of these observations are given in Section 2. In Section 3, we outline methods used to model the dust emission, which allow us to constrain their physical properties, including dust mass, temperature, and infrared luminosity. Next, we present the results of this analysis in Section 4, focusing on the four spatially extended $\mathrm{H}$ II regions $(\mathrm{H} 1, \mathrm{H} 2, \mathrm{H} 3$, and $\mathrm{H} 5)$. We compare the FORCAST data to archival maps of the H II regions at near-, mid-, and far-infrared wavelengths to better understand their morphology. We also examine the spatial distribution of dust temperatures in these objects to determine whether they are consistent with being heated by a single star or whether multiple sources are needed to reproduce the observations. In Section 5, we discuss the possible heating sources for each of the $\mathrm{H}$ II regions and identify possible stellar counterparts. Additionally, we consider possible origins for these stars and examine whether any of the $\mathrm{H}$ II regions have infrared luminosities that could be indicative of a small embedded stellar cluster. Lastly, we discuss a collection of newly identified infrared sources that may be associated with the $\mathrm{H}$ complex.

\section{Observations and Data Reduction}

\subsection{SOFIA/FORCAST}

We observed the $\mathrm{H} \mathrm{H}$ II regions with the $2.5 \mathrm{~m}$ telescope aboard the Stratospheric Observatory for Infrared Astronomy (SOFIA; Young et al. 2012) using the FORCAST instrument (Herter et al. 2012). FORCAST is a $256 \times 256$ pixel dualchannel, wide-field mid-infrared camera with a field of view of $3 ! 4 \times 3 ! 2$ and a plate scale of $0 ! 768$ pixel $^{-1}$. The two channels consist of a short-wavelength camera operating at 5-25 $\mu \mathrm{m}$ and a long-wavelength camera operating at $28-40 \mu \mathrm{m}$. An internal dichroic beam splitter enables simultaneous observation with both cameras, while a series of bandpass filters are used to select specific wavelengths.

The two fields containing the $\mathrm{H} \mathrm{H}$ II regions were observed during SOFIA cycle 3 on 2015 July 4 during flight number 225 (Program ID: 70_0300; PI: T. Herter). Both fields were observed with the $19.7,25.2,31.5$, and $37.1 \mu \mathrm{m}$ filters. A fivepoint dither pattern was used to remove bad pixels and mitigate response variations. Parallel chopping and nodding on the array were used to remove the sky and telescope thermal backgrounds. The frequency of the chop throw was $\sim 4 \mathrm{~Hz}$, and the integration time at each nod position was $\sim 23 \mathrm{~s}$.
Table 1

Observation Details

\begin{tabular}{|c|c|c|c|c|}
\hline Field & $\begin{array}{c}\text { Filter } \\
1(\mu \mathrm{m})\end{array}$ & $\begin{array}{c}\text { Filter } \\
2(\mu \mathrm{m})\end{array}$ & Integration (s) & $\begin{array}{l}\text { Aircraft } \\
\text { Alt. (feet) }\end{array}$ \\
\hline H North & 19.7 & 31.5 & 136 & $\sim 36,000$ \\
\hline H North & 25.2 & 31.5 & 119 & $\sim 36,000$ \\
\hline H North & 25.2 & 37.1 & 345 & $\sim 36,000$ \\
\hline H South & 19.7 & 31.5 & 46.3 & $\sim 40,000$ \\
\hline H South & 25.2 & 31.5 & 37.8 & $\sim 40,000$ \\
\hline H South & 25.2 & 37.1 & 84.1 & $\sim 40,000$ \\
\hline
\end{tabular}

Note. All observations were obtained during SOFIA cycle 3 during flight 225 on 2015 July 4

The observations were processed using the pipeline steps described in Herter et al. (2013). The processed data products were downloaded from the SOFIA Data Cycle System. ${ }^{6}$ The quality of the co-added images is consistent with neardiffraction-limited imaging from 19.7 to $37.1 \mu \mathrm{m}$. The FWHM of the point-spread function (PSF) was 3."3 at $19.7 \mu \mathrm{m}, 3 . " 2$ at $25.2 \mu \mathrm{m}, 3 . .5$ at $31.5 \mu \mathrm{m}$, and $3 . " 9$ at $37.1 \mu \mathrm{m}$. The rms noise per pixel was $9.7 \mathrm{mJy}$ at $19.7 \mu \mathrm{m}, 18 \mathrm{mJy}$ at $25.2 \mu \mathrm{m}, 21 \mathrm{mJy}$ at $31.5 \mu \mathrm{m}$, and $18 \mathrm{mJy}$ at $37.1 \mu \mathrm{m}$. The estimated $3 \sigma$ uncertainty in the flux calibration is $\pm 20 \%$. Table 1 provides additional details on the FORCAST observations.

In order to produce a larger image mosaic of the surrounding region, we included FORCAST observations of the Arched Filaments H II region that cover the location of the Arches cluster. These data were originally presented in Hankins et al. (2017), and details of these observations can be found therein. Additional post-processing was performed on all of the FORCAST data to bring the maps at different wavelengths to the same angular resolution. The data were deconvolved using the Richardson-Lucy algorithm (Richardson 1972; Lucy 1974), and the PSF was estimated using a Gaussian kernel corresponding to the beam size at the given wavelength. Lastly, the maps were convolved back to a uniform beam size of $4 . \prime 0$. Figure 1 displays a false-color map of of the $\mathrm{H}$ II regions using the $19.7,25.2$, and $37.1 \mu \mathrm{m}$ data.

\subsection{Data from Other Sources}

Archival data from several sources were incorporated into our study of the $\mathrm{H} \mathrm{H}$ II regions. Maps from the HST Pa $\alpha$ survey of the GC (Wang et al. 2010) were used to examine ionized gas emission and near-infrared continuum sources in the region. Similarly, observations from the GLIMPSE Spitzer/IRAC survey (Churchwell et al. 2009) were used to examine the morphology of the $\mathrm{HII}$ regions and also to expand the wavelength coverage of the spectral energy distributions (SEDs) for the sources. We also reference maps of the H II regions at far-infrared wavelengths that were observed as a part of the Herschel Hi-GAL survey (Molinari et al. 2010). In addition to the archival observations, we utilized source catalogs from the HST Pa $\alpha$ survey of the GC (Dong et al. 2011), the Two Micron All Sky Survey (2MASS; Cutri et al. 2003), and the Spitzer IRAC survey of the GC (Ramírez et al. 2008) to examine associations between sources in each of the data sets.

\footnotetext{
6 https://dcs.sofia.usra.edu/
} 


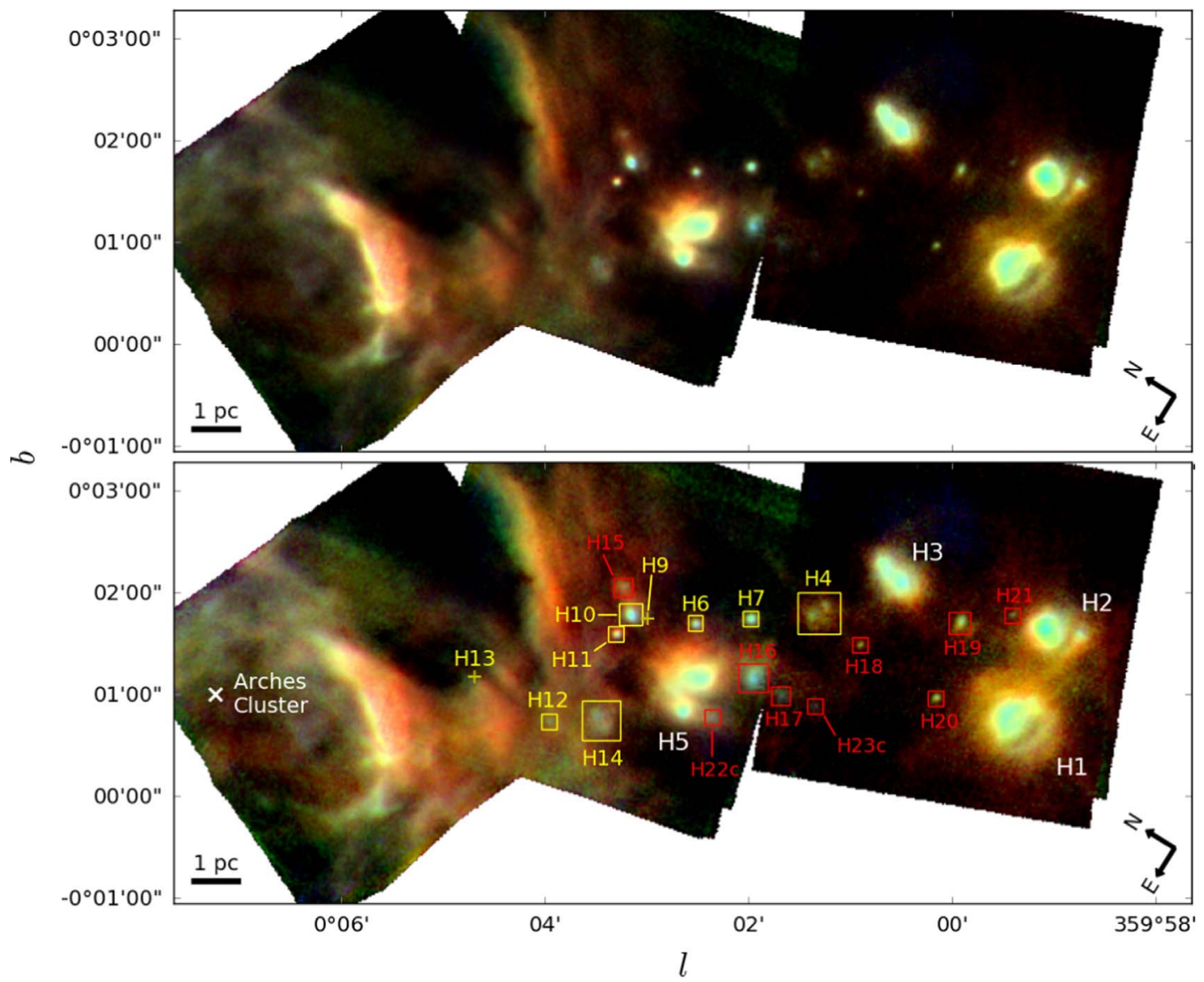

Figure 1. Top: false-color map of the H H II regions created with SOFIA/FORCAST observations at 19.7 (blue), 25.2 (green), and $37.1 \mu \mathrm{m}$ (red). Bottom: second RGB map created with the same data at a different stretch. Sources within the map are grouped into three categories that are indicated by the color of their labels. The spatially extended $\mathrm{H}$ II regions $(\mathrm{H} 1, \mathrm{H} 2, \mathrm{H} 3$, and $\mathrm{H} 5)$ are shown in white. Additional $\mathrm{H}$ II regions in the complex, including H4, H6, H7, H10, H1 1, H12, and H14, are shown in yellow. The known sources $\mathrm{H} 9$ and $\mathrm{H} 13$ are not detected by FORCAST, though their positions are indicated with a plus sign. A number of additional infrared sources (H15-H23) were also identified in the maps and are shown in red. Two of the sources (H22c and H23c) are listed as candidates because they are only detected in a single FORCAST band. The white cross denotes the location of the Arches cluster.

\subsection{Extinction Correction}

Objects near the GC suffer significant extinction due to large column densities of gas and dust along the line of sight $\left(A_{V} \sim 30\right.$; Cardelli et al. 1989). In this work, we adopt the extinction law of Fritz et al. (2011) with $A_{K_{s}}=2.58$ to deredden the infrared fluxes of the observed sources. The Fritz law was derived from hydrogen recombination line observations of Sgr A West and has been adopted in our previous studies of objects in the GC. A handful of stars associated with the $\mathrm{H} \mathrm{H}$ II regions ${ }^{7}$ show a range of near-infrared extinction $\left(A_{K} \approx 2.0-3.2\right.$; Dong et al. 2015). While correcting for this range of extinction values is troublesome at near-infrared wavelengths, the extinction becomes considerably less at longer wavelengths where FORCAST operates (e.g., $A_{1.9} / A_{25.2} \approx 10$ ). Therefore, the measured fluxes from FORCAST are more robust to modest extinction variations, which could be present across the field.

\section{Analysis}

In this section, we describe our methodology for studying the infrared sources detected by FORCAST in the H complex. Our primary focus is on the four $\mathrm{HII}$ regions $\mathrm{H} 1, \mathrm{H} 2, \mathrm{H} 3$, and $\mathrm{H} 5$. These objects are spatially extended and sufficiently bright to

Including P35, P114, and P107. study their morphologies and make comparisons with data at other wavelengths. We also use the FORCAST data to analyze the spatial distribution of dust temperatures in these objects to investigate the heating sources contained within. Lastly, we model infrared SEDs of these objects along with other detected H II regions in the complex, including $\mathrm{H} 4, \mathrm{H} 6, \mathrm{H} 7, \mathrm{H} 10, \mathrm{H} 11$, and H14. Results for each of these sources can be found in Section 4. In addition to the known $\mathrm{HII}$ regions, several other infrared sources were also detected in the complex (H15-H23c; Figure 1). These newly identified sources are discussed in Section 5.

\subsection{Multiwavelength Morphology}

Morphological differences in multiwavelength imaging can help to distinguish between various emission components in and around the $\mathrm{H}$ II regions. For example, previous work with SOFIA/FORCAST imaging observations has demonstrated the utility of 19.7 and $37.1 \mu \mathrm{m}$ emission for differentiating between dust emission associated with $\mathrm{H}$ II regions and the surrounding photodissociation regions (e.g., Salgado et al. 2016). Utilizing this information aids in our understanding of the structure of the regions and can help to localize the dust-heating and ionization sources. In this study, we compare the SOFIA/ FORCAST observations to archival maps of the region from 1.9 to $160 \mu \mathrm{m}$ (see Section 2.2 for the complete listing). We use these data to analyze the morphology of individual $\mathrm{H}$ II regions 
at near-, mid-, and far-infrared wavelengths, while also examining the relationship between the dust emission and ionized gas emission (via $\mathrm{Pa} \alpha$ ). Multiwavelength maps of the objects $\mathrm{H} 1, \mathrm{H} 2, \mathrm{H} 3$, and $\mathrm{H} 5$ are presented in Section 4, along with a description of their morphology.

\subsection{Color-Temperature and Optical Depth Maps}

We produce color-temperature maps of the $\mathrm{H}$ II regions using the 19.7 and $37.1 \mu \mathrm{m}$ data. This is accomplished by expressing the frequency dependence of the emission as $F_{\nu} \propto \nu^{\beta} B_{\nu}\left(T_{D}\right)$, where $T_{D}$ is the color-temperature and the dust emissivity is taken to be of the form $\nu^{\beta}$ with $\beta=2$. For optically thin emission, which is virtually always the case (see below), $T_{D}$ represents an average dust temperature along a line of sight weighted by both temperature and density.

We investigate the source(s) responsible for heating dust in the H II regions by calculating the expected dust temperature based on the properties of the heating source and comparing this with the observed color temperature. For a dust grain in thermal equilibrium with an incident radiation field, the temperature is determined by the optical properties of the grain and the local radiation field. If the dust is heated by a source with luminosity, $L$, at a distance, $d$, the equilibrium temperature of the dust, $T_{d}$, is given by

$$
T_{d}=\left(\frac{L}{16 \pi \sigma d^{2}} \frac{Q_{\mathrm{UV}}}{Q_{d}^{\prime}}\right)^{1 /(4+\beta)},
$$

where $Q_{\mathrm{UV}}$ is the grain absorption cross section averaged over the spectrum of the incident radiation field and $Q^{\prime}{ }_{d}$ is a temperature-independent dust emission efficiency of the form $Q_{d}^{\prime}=\left\langle Q_{\text {abs }}\right\rangle(T / K)^{-2}=1.3 \times 10^{-6}(a / 0.1 \mu \mathrm{m})$, which follows from the expression for silicate grains from Draine (2011). Writing $Q^{\prime}{ }_{d}$ in this fashion allows us to solve for the grain temperature as it is shown in Equation (1). In this expression, the grain size plays a substantial role in setting the equilibrium dust temperature, and we adopt a characteristic grain radius of $a=0.01 \mu \mathrm{m}$. This value is smaller than typical ISM dust grains $(a \approx 0.1 \mu \mathrm{m})$; however, it is consistent with previous determinations of dust grain characteristics for $\mathrm{H}$ II regions in the GC (e.g., Lau et al. 2016; Hankins et al. 2017).

We calculate temperature profiles for dust in the $\mathrm{H}$ II regions according to Equation (1), assuming that the candidate ionization source (if known) is also the dust-heating source. To account for projection effects, we assume that the true distance between the heating source and the dust is approximately $\sqrt{2}$ times the projected distance. This procedure provides an estimate of the maximum temperature that can be achieved at a given distance from the supposed heating source. Comparing the calculated temperatures with the observed temperatures can reveal locations that are warmer than expected, which implies some contribution from an additional heating source. ${ }^{8}$

\footnotetext{
8 Adopting dust grains with a characteristic radius $a=0.01 \mu \mathrm{m}$ rather than a more typical value for ISM grains $(0.1 \mu \mathrm{m})$ increases the expected equilibrium temperature by $\sim 50 \%$ for a given distance. In cases where we claim that additional heating sources are needed, our grain size assumption does not adversely affect this result because the larger grain size would require the equilibrium distance of the dust grain be a factor of $\sim 3$ smaller in order to achieve the same temperature as the $0.01 \mu \mathrm{m}$ sized grain. Thus, larger grains would only strengthen our argument in favor of additional heating sources.
}

In addition to temperature information, the FORCAST data can also be used to infer the optical depth of the dust. If the dust emission is optically thin, then the optical depth can be expressed as

$$
\tau_{\lambda}=\frac{F_{\lambda}}{\Omega_{p} B_{\nu}\left(T_{d}\right)},
$$

where $F_{\lambda}$ is the flux per pixel, $\Omega_{p}$ is the solid angle subtended by each pixel, and $B_{\nu}\left(T_{d}\right)$ is the Planck function at the dust temperature, $T_{d}$. The calculated opacities of the regions verify that the emission is optically thin, typically $\tau_{37}<0.01$. Uncertainties in the color temperature and optical depth values are typically $\sim 15 \%$ and $\sim 25 \%$, respectively. However, one must be mindful of the fact that line-of-sight effects can influence both temperature and column density measurements. In some cases this can lead to artificial minima and maxima in the column density, which are heavily influenced by minima and maxima in the dust temperature. Maps of the color temperature and the optical depth for the extended H II regions can be found in Section 4.

\subsection{SED Models with DustEM}

SEDs for each of the $\mathrm{H}$ II regions were constructed using data from the four Spitzer/IRAC bands (3.6, 4.5, 5.8, and $8.0 \mu \mathrm{m})$, the four SOFIA/FORCAST bands $(19.7,25.2,31.5$, and $37.1 \mu \mathrm{m})$, and the Herschel/PACS $70 \mu \mathrm{m}$ data. ${ }^{9}$ Fluxes for the sources were extracted using aperture photometry. The extinction-corrected FORCAST fluxes are listed in Table 2, along with information on the apertures used for both extended and point sources. To minimize contamination in the apertures, we fit and subtract away unrelated stellar sources using a Gaussian PSF model. This is especially important for extended sources in the shortest-wavelength IRAC bands, which would otherwise be dominated by bright point sources. Aperture photometry extracted from the IRAC data was corrected for the aperture size in accordance with the instrument handbook. ${ }^{10}$ Fluxes for point-like sources were also checked against the Spitzer/IRAC GC catalog from Ramírez et al. (2008) for consistency. In the few $\mathrm{H}$ II regions where a candidate ionizing source has been previously identified, we also include photometry of the star from the HST F190 continuum observations.

Dust emission models of the $\mathrm{H}$ II regions were generated using DustEM (Compiègne et al. 2011). DustEM incorporates transient heating of grains and is therefore able to model the emission from stochastically heated grains, such as polycyclic aromatic hydrocarbons (PAHs). By modeling the dust emission from each source, we are able to study any differences in their physical properties, such as the strength of the local radiation field and the abundances of certain types of grains. The adopted and fitted model parameters are discussed in the next subsection.

\subsubsection{Model Parameters}

The interstellar radiation field, $G_{0}$, from Mathis et al. (1983) scaled by an overall intensity factor was used as the input

\footnotetext{
The $160 \mu \mathrm{m}$ data were not used in the SEDs because they appear to be significantly contaminated by background molecular clouds.

${ }^{10}$ https://irsa.ipac.caltech.edu/data/SPITZER/docs/irac/iracinstrumenthand book/27/
} 
Table 2

Dereddened Source Fluxes

\begin{tabular}{|c|c|c|c|c|c|c|c|c|c|c|}
\hline Source & $F_{3.6}(\mathrm{Jy})$ & $F_{4.5}(\mathrm{Jy})$ & $F_{5.8}(\mathrm{Jy})$ & $F_{8.0}(\mathrm{Jy})$ & $F_{19.7}(\mathrm{Jy})$ & $F_{25.2}(\mathrm{Jy})$ & $F_{31.5}(\mathrm{Jy})$ & $F_{37.1}(\mathrm{Jy})$ & $F_{70}(\mathrm{Jy})$ & $R_{a p}(\operatorname{arcsec})^{\mathrm{a}}$ \\
\hline$\overline{\mathrm{H} 1}$ & $9.27 \pm 6.50$ & $8.92 \pm 5.35$ & $11.6 \pm 4.6$ & $36.7 \pm 11$ & $1420 \pm 430$ & $2030 \pm 610$ & $1680 \pm 500$ & $1560 \pm 470$ & $598 \pm 167$ & 23 \\
\hline $\mathrm{H} 2$ & $8.16 \pm 5.61$ & $9.65 \pm 5.11$ & $18.6 \pm 6.4$ & $73.4 \pm 16.1$ & $1840 \pm 370$ & $1950 \pm 390$ & $1420 \pm 280$ & $1240 \pm 250$ & $390 \pm 112$ & 20 \\
\hline H3 & $2.86 \pm 3.27$ & $2.74 \pm 2.65$ & $6.35 \pm 3.49$ & $23.9 \pm 7.6$ & $779 \pm 156$ & $1050 \pm 210$ & $915 \pm 183$ & $815 \pm 163$ & $360 \pm 108$ & 21 \\
\hline H4 & $2.40 \pm 3.05$ & $1.56 \pm 2.05$ & $2.92 \pm 2.63$ & $6.03 \pm 4.04$ & $45.7 \pm 9.1$ & $110 \pm 22$ & $124 \pm 25$ & $118 \pm 24$ & $39.3 \pm 28.8$ & 12 \\
\hline H5 & $9.29 \pm 6.01$ & $8.26 \pm 4.73$ & $18.8 \pm 6.4$ & $53.2 \pm 12.8$ & $1270 \pm 250$ & $1920 \pm 380$ & $1770 \pm 350$ & $1840 \pm 370$ & $792 \pm 242$ & 25 \\
\hline H6 & $0.0929 \pm 0.0232$ & $0.119 \pm 0.030$ & $0.266 \pm 0.066$ & $1.42 \pm 0.35$ & $22.6 \pm 5.7$ & $28.9 \pm 7.2$ & $25.8 \pm 6.5$ & $24.7 \pm 6.2$ & $4.73^{\mathrm{b}}$ & 6 \\
\hline $\mathrm{H} 7$ & $0.205 \pm 0.051$ & $0.265 \pm 0.066$ & $0.520 \pm 0.130$ & $1.70 \pm 0.42$ & $33.9 \pm 8.5$ & $44.8 \pm 11.2$ & $38.9 \pm 9.7$ & $36.9 \pm 9.1$ & $11.8^{\mathrm{b}}$ & 6 \\
\hline $\mathrm{H} 10$ & $0.353 \pm 0.106$ & $0.396 \pm 0.119$ & $0.853 \pm 0.256$ & $3.18 \pm 0.95$ & $71.7 \pm 21.5$ & $82.8 \pm 24.8$ & $76.5 \pm 22.9$ & $71.6 \pm 21.4$ & $13.2^{\mathrm{b}}$ & 8 \\
\hline H11 & $0.0451 \pm 0.0136$ & $0.0614 \pm 0.0184$ & $0.222 \pm 0.067$ & $0.660 \pm 0.198$ & $16.6 \pm 3.3$ & $25.2 \pm 5.1$ & $22.5 \pm 4.5$ & $24.8 \pm 5.0 x$ & $22.7 \pm 6.8$ & 6 \\
\hline H14 & $0.860 \pm 0.0258$ & $0.692 \pm 0.207$ & $2.20 \pm 0.66$ & $8.43 \pm 2.53$ & $60.2 \pm 23.6$ & $66.2 \pm 23.9$ & $50.2 \pm 17.9$ & $38.4 \pm 14.0$ & $29.1 \pm 17.8$ & 11 \\
\hline
\end{tabular}

Notes.

a The radius of the circular aperture used in the photometric extraction for the sources. For unresolved sources $R_{a p}=6^{\prime \prime}$.

${ }^{\mathrm{b}}$ Upper limits on the $70 \mu \mathrm{m}$ flux values. 
Table 3

Best-fit DustEM Model Parameters

\begin{tabular}{lccccc}
\hline \hline Region & Alternate ID & Radiation Field $\left(G_{0}\right)$ & $X_{\text {PAH }}$ & $L_{\text {IR }}\left(L_{\odot}\right)$ & Dust Mass $\left(M_{\odot}\right)$ \\
\hline H1 & G359.990+00.013 & $3.0_{-2.0}^{+1.0} \times 10^{4}$ & $0.2_{-0.15}^{+0.15}$ & $5.2_{-1.3}^{+1.1} \times 10^{5}$ & $1.2_{-0.25}^{+0.3} \times 10^{-1}$ \\
H2 & G359.985+00.027 & $5.0_{-2.5}^{+4.0} \times 10^{4}$ & $0.5_{-0.2}^{+0.2}$ & $5.0_{-1.5}^{+0.9} \times 10^{5}$ & $5.9_{-1.6}^{+1.2} \times 10^{-2}$ \\
H3 & G00.0095+00.036 & $2.0_{-1.0}^{+3.0} \times 10^{4}$ & $0.3_{-0.25}^{+0.25}$ & $3.0_{-0.8}^{+0.5} \times 10^{5}$ & $9.2_{-2.4}^{+1.4} \times 10^{-2}$ \\
H5 & G000.042+00.017 & $1.0_{-0.3}^{+1.5} \times 10^{4}$ & $0.5_{-0.25}^{+0.25}$ & $5.7_{-1.5}^{+0.8} \times 10^{5}$ & $3.4_{-0.9}^{+0.5} \times 10^{-1}$ \\
\hline H4 & G000.021+00.029 & $6.0_{-3.0}^{+4.0} \times 10^{3}$ & $1.0_{-0.7}^{+0.8}$ & $3.5_{-1.0}^{+0.7} \times 10^{4}$ & 0.9 \\
H6 & G000.042+00.028 & $4.0_{-1.5}^{+2.5} \times 10^{4}$ & $0.5_{-0.3}^{+0.2}$ & $8.3_{-1.5}^{+1.8} \times 10^{3}$ & $3.5_{-1.0}^{+0.6} \times 10^{-2}$ \\
H7 & G000.033+00.029 & $4.0_{-3.0}^{+2.5} \times 10^{4}$ & $0.55_{-0.20}^{+0.25}$ & $1.3_{-0.3}^{+0.3} \times 10^{4}$ & $1.3_{-0.3}^{+0.3} \times 10^{-3}$ \\
H10 & G000.053+00.030 & $3.0_{-1.5}^{+5.0} \times 10^{4}$ & $0.70_{-0.30}^{+0.25}$ & $2.2_{-0.6}^{+0.6} \times 10^{4}$ & $2.0_{-0.5}^{+0.3} \times 10^{-3}$ \\
H11 & G000.055+00.026 & $3.0_{-1.5}^{+3.0} \times 10^{4}$ & $1.0_{-0.3}^{+0.6}$ & $3.2_{-1.0}^{+1.1} \times 10^{3}$ & 2.5 \\
H14 & G000.058+00.013 & $4.0_{-3.0}^{+5.0} \times 10^{4}$ & 1.3 & $6.2_{-2.2}^{+2.3} \times 10^{-3}$ & 3.2 \\
& & & $1.6_{-0.6}^{+0.5}$ & $2.0_{-0.4}^{+0.5} \times 10^{4}$ & 2.2 \\
\end{tabular}

radiation field for the models. Values for the radiation field in the models range from $\sim 10^{3} G_{0}$ to $10^{4} G_{0}$, which are fairly typical for H II regions (Tielens \& Hollenbach 1985). For dust properties, we adopt abundances for the diffuse ISM from Compiègne et al. (2011), which consists of four grain species with the following mass fractions: ${ }^{11}$ PAHs $\left(Y_{\mathrm{PAH}}=7.8 \times 10^{-4}\right)$, small amorphous carbon $\left(\mathrm{SamC} ; Y_{\mathrm{SamC}}=1.65 \times 10^{-4}\right)$, large amorphous carbon (LamC; $\left.Y_{\text {LamC }}=1.45 \times 10^{-4}\right)$, and astronomical silicates $\left(\mathrm{aSil} ; \quad Y_{\mathrm{aSil}}=7.8 \times 10^{-3}\right)$. Mid-infrared emission features from PAHs dominate the Spitzer/IRAC bands, which permit the PAH component to be fitted separately from the other dust species whose abundances were left fixed in the models. We denote the relative change in PAH abundance compared to the diffuse ISM value as a multiplicative factor $X_{\mathrm{PAH}}$. Allowing the modeled PAH abundance to vary improves the quality of the SED fits; however, there are caveats with interpreting these models as a true change in PAH abundance because the emitting regions for the dust components can be different, especially if they originate from inside the $\mathrm{H}$ II region or photodissociation region (e.g., Salgado et al. 2016). This may introduce a volume filling factor that complicates the true abundance ratio of the different dust components.

In total, three parameters are fit for each model: the intensity of the input radiation field, the relative PAH abundance, and the overall scaling of the SED model (which is related to the infrared luminosity and dust mass). The best-fit model parameters are determined by constructing a grid of models and calculating the reduced $\chi^{2}$ for each set of parameters. Details on the best-fit models for each of the $\mathrm{H}$ II regions can be found in Table 3.

\section{Results}

\section{1. $H 1$}

$\mathrm{H} 1$ is located at $l=359^{\circ} 59^{\prime} 20^{\prime \prime}, b=00^{\circ} 00^{\prime} 42^{\prime \prime}$, making it one of the southernmost sources in the $\mathrm{H}$ complex (Figure 1). Its ionizing source is thought to be the known emission-line star P114, which is classified as an O4-6I subtype (Dong et al. 2015). In Figure 2, we present multiwavelength imaging of H1 along with the color-temperature and optical depth maps of the source. Thermal dust emission from $\mathrm{H} 1$ shows a bright arc-like structure, which is indicative of an interaction between the star and the surrounding ISM. This feature could be generated by a bow shock as the star passes through intervening material, or it

\footnotetext{
11 The stated mass fractions of the components are expressed relative to the mass of hydrogen (i.e., $Y_{A}=M_{A} / M_{\mathrm{H}}$, where $A$ is one of the dust species).
}

could be a pressure-driven "blister" caused by a large density gradient in the star's surroundings (Dong et al. 2015). At FORCAST wavelengths, the arc-like structure is the brightest component of the emission, though there appears to be a continuation of the feature that completes a nearly circular shape (with a diameter of $\sim 0.9 \mathrm{pc}$, though P114 is offset from the center). The bubble-like appearance of $\mathrm{H} 1$ provides possible support for the "blister" H II region scenario for this object. This possibility is discussed further in Section 5.1.

Color-temperature analysis of $\mathrm{H} 1$ (Figure 2) shows that P114 is consistent with being the only heating source for dust in the region. The hottest dust $(130 \pm 20 \mathrm{~K})$ is coincident with the location of the bright dust arc observed in the infrared continuum maps. The temperature of this dust suggests that this structure is physically closer to P114 than other parts of the H II region.

The $37 \mu \mathrm{m}$ optical depth of $\mathrm{H} 1$ ranges between $2.2 \times 10^{-3}$ and $7.4 \times 10^{-3}$. There is evidence for a slight increase in the dust column at the position of the brightest $37 \mu \mathrm{m}$ emission (shown in contours in Figure 2). The largest opacities found along the edge of the map toward the north and east are in the direction of the molecular cloud that P114 is interacting with. However, these edge features should be viewed with caution because they are near the signal-to-noise cutoffs for the data.

The SED model of H1 (Figure 6) is best fit by a source with an infrared luminosity of $L_{\mathrm{IR}}=5.2_{-1.3}^{+1.1} \times 10^{5} L_{\odot}$ and dust mass $M_{d}=1.2_{-0.25}^{+0.3} \times 10^{-1} M_{\odot}$. The dust model from Compiègne et al. (2011) reliably reproduces the observed emission; however, the PAH abundance is best fit with $X_{\text {PAH }}=0.2_{-0.15}^{+0.15}$. The reduced- $\chi^{2}$ value of the best-fit model is 1.9. Further information on the SED model can be found in Table 3.

For completeness, we also include a model of stellar flux of P114 (light-blue dashed line). Based on its measured flux in the F190 filter, we do not expect that P114 contributes significantly to the total flux beyond the near-infrared bands. As a consistency check, we compared the stellar luminosity of P114 and the infrared luminosity of the H II region. If P114 is the only heating source in $\mathrm{H} 1$, then the luminosity of the star should be greater than or equal to that of H1. As noted earlier, P114 is classified as an O4-6I subtype, for which Martins et al. (2005) provide a range of luminosities between $6.0 \times 10^{5}$ and $8.7 \times 10^{5} L_{\odot}$. These values are comparable to the measured infrared luminosity of $\mathrm{H} 1 \quad\left(L_{\mathrm{IR}}=5.2_{-1.3}^{+1.1} \times 10^{5} L_{\odot}\right)$, which implies a dust covering fraction $\left(L_{\mathrm{IR}} / L_{\star}\right)$ between $\sim 0.85$ and 0.60 . 

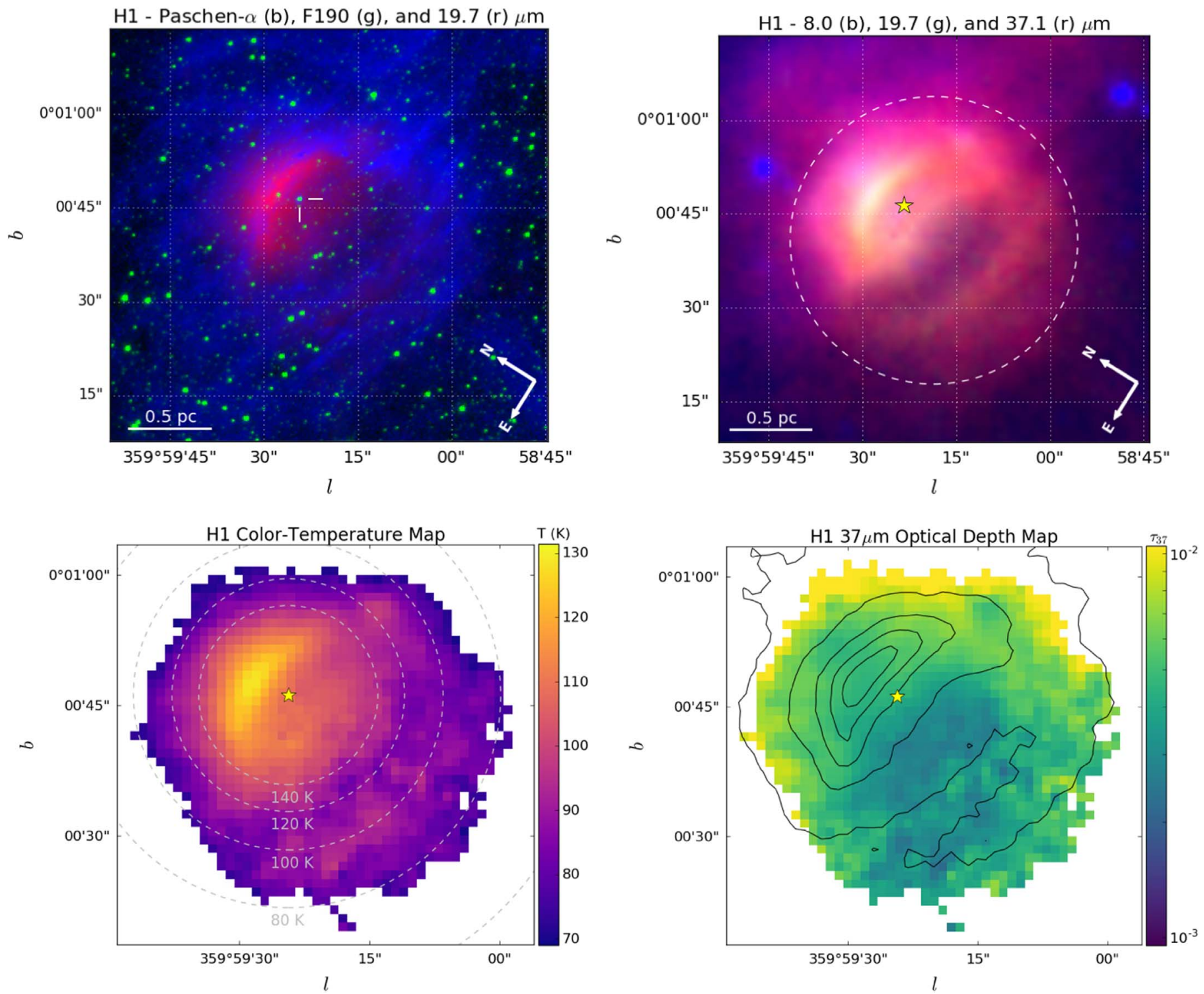

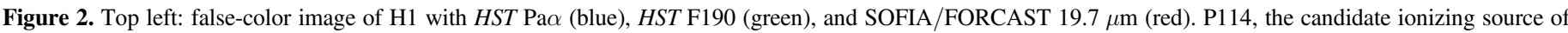

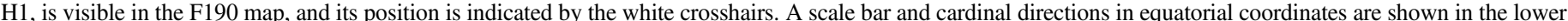

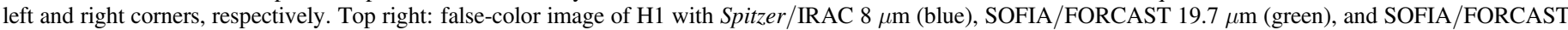

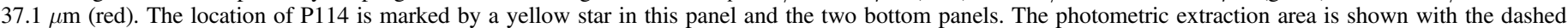

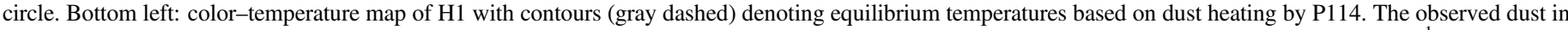

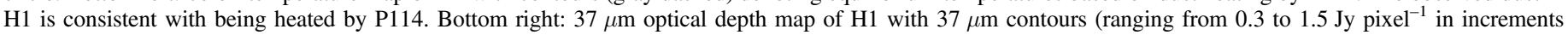
of 0.3 ).

\section{2. $H 2$}

$\mathrm{H} 2$ is located in the southern part of the $\mathrm{H}$ complex and is adjacent to $\mathrm{H} 1$ (Figure 1). The ionizing source of $\mathrm{H} 2$ is thought to be the known emission-line star P35, which is classified as type $\mathrm{O} \mathrm{If}^{+}$(Dong et al. 2015). In Figure 3, we present multiwavelength imaging of $\mathrm{H} 2$, along with the colortemperature and optical depth maps of this object. The midinfrared maps of $\mathrm{H} 2$ show evidence for two components that we refer to as H2-Northeast and H2-Southwest (henceforth $\mathrm{H} 2 \mathrm{NE}$ and $\mathrm{H} 2 \mathrm{SW}){ }^{12} \mathrm{H} 2 \mathrm{NE}$ is coincident with the location of $\mathrm{P} 35$, while $\mathrm{H} 2 \mathrm{SW}$ is located $\sim 20^{\prime \prime}$ to the southwest. H2NE is the highest surface brightness object of any of the sources in

\footnotetext{
${ }^{12}$ Following the naming scheme of Zhao et al. (1993), who named the components of $\mathrm{H} 5$ following from their relative positions in equatorial coordinates rather than Galactic coordinates.
}

the $\mathrm{H}$ complex at FORCAST wavelengths. Its emission is spatially resolved by FORCAST $\left(\mathrm{FWHM}_{19.7}=7\right.$ !" $7 \times 6$ ". 6 , $\mathrm{FWHM}_{37.1}=12^{\prime \prime} \times 9$ !' 8 ), though there does not appear to be significant structure to this object other than the bright, centrally peaked source located near P35.

$\mathrm{H} 2 \mathrm{SW}$ appears to be located at the end of a filament of ionized gas and dust, which is visible in the $\mathrm{Pa} \alpha$ data, as well as the four FORCAST bands. Whether these structures actually are associated is unclear, but their spatial coincidence is somewhat suggestive. This filamentary structure was noted in previous radio mapping of the region by Yusef-Zadeh et al. (2004) and appears to run from the top of H1 to its termination at the location of $\mathrm{H} 2 \mathrm{SW}$.

Color-temperature analysis of $\mathrm{H} 2$ shows that $\mathrm{P} 35$ is consistent with being the heating source for H2NE; however, $\mathrm{H} 2 \mathrm{SW}$ must be independently heated. We will investigate the 

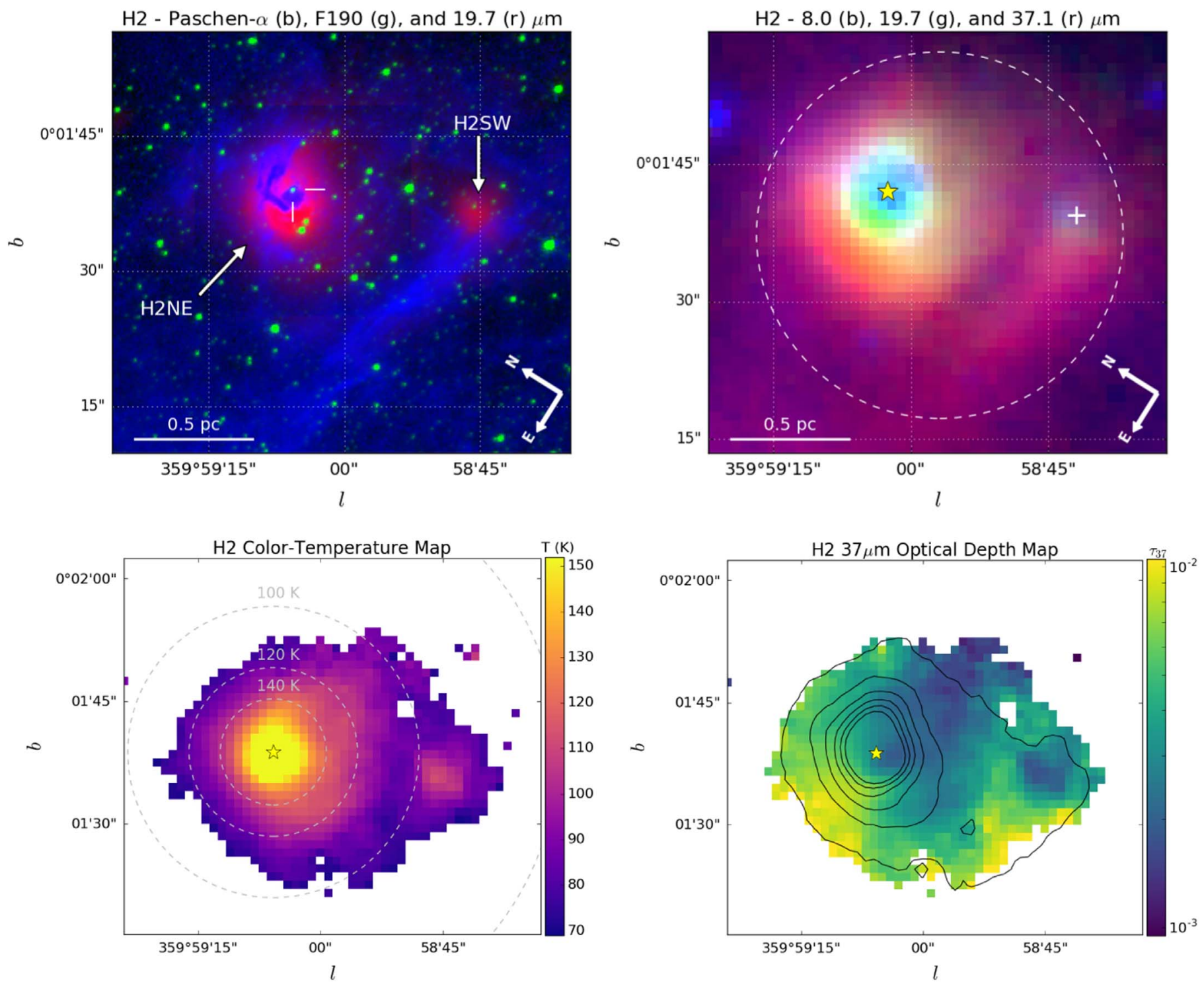

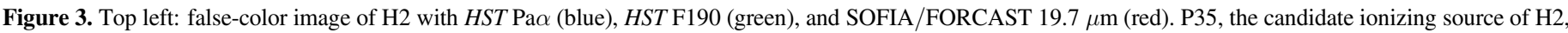

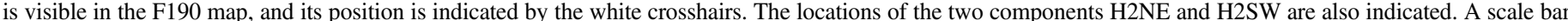

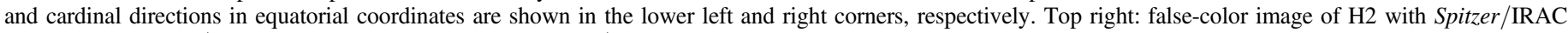

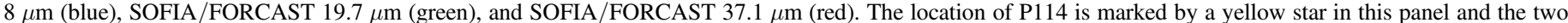

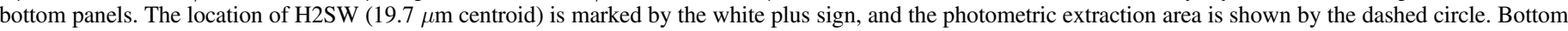

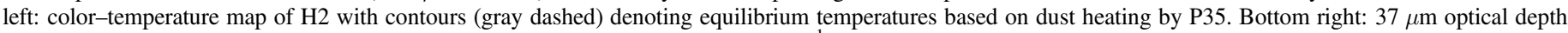

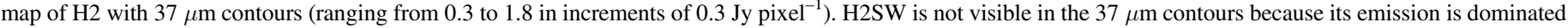
by an extended dust filament.

heating source of $\mathrm{H} 2 \mathrm{SW}$ in greater detail in Section 4.6.1. Near the location of P35, the peak dust temperature is $210 \pm 32 \mathrm{~K}$. This is significantly warmer than any of the other $\mathrm{H}$ II regions in the complex.

The $37 \mu \mathrm{m}$ optical depth of $\mathrm{H} 2$ ranges between $3.6 \times 10^{-4}$ and $9.8 \times 10^{-3}$. The calculated optical depth at the location of $\mathrm{H} 2 \mathrm{SW}$ is a local minimum, which is somewhat surprising if this is a heavily embedded source. However, as noted in Section 3.2, line-of-sight effects could influence the measured optical depth because the determination is a strong function of temperature. A more detailed model of this source is needed to characterize its physical properties.

The SED model of $\mathrm{H} 2$ is best fit by a source having an infrared luminosity of $L_{\mathrm{IR}}=5.0_{-1.5}^{+0.9} \times 10^{5} L_{\odot}$ and dust mass $M_{d}=5.9_{-1.6}^{+1.2} \times 10^{-2} M_{\odot}$. Similar to the case of $\mathrm{H} 1$, the adopted dust abundances from Compiègne et al. (2011) reliably reproduce the observed emission, though the PAH abundance is best fit by $X_{\mathrm{PAH}}=0.5_{-0.2}^{+0.2}$. The reduced- $\chi^{2}$ value of the best-fit model is 1.8. We also include a model of the stellar flux of P35 in the SED (light-blue dashed line). Based on its flux in the F190 filter, we do not expect that P35 contributes directly to the total flux beyond the near-infrared bands.

If $\mathrm{P} 35$ is the primary heating source of $\mathrm{H} 2 \mathrm{NE}$, which dominates the total luminosity of $\mathrm{H} 2$, then we would expect the luminosity of the star to be greater than or equal to the infrared luminosity of the $\mathrm{H}$ II region. Because the subtype of P35 is not well constrained, the temperature, and therefore the luminosity, of the star could span a relatively large range of values $\left(3.2 \times 10^{5}-1 \times 10^{6} \mathrm{~L}_{\odot}\right.$; Martins et al. 2005). The constraint supplied by the infrared luminosity implies that P35 is an $\mathrm{O} 7$ or earlier subtype. 

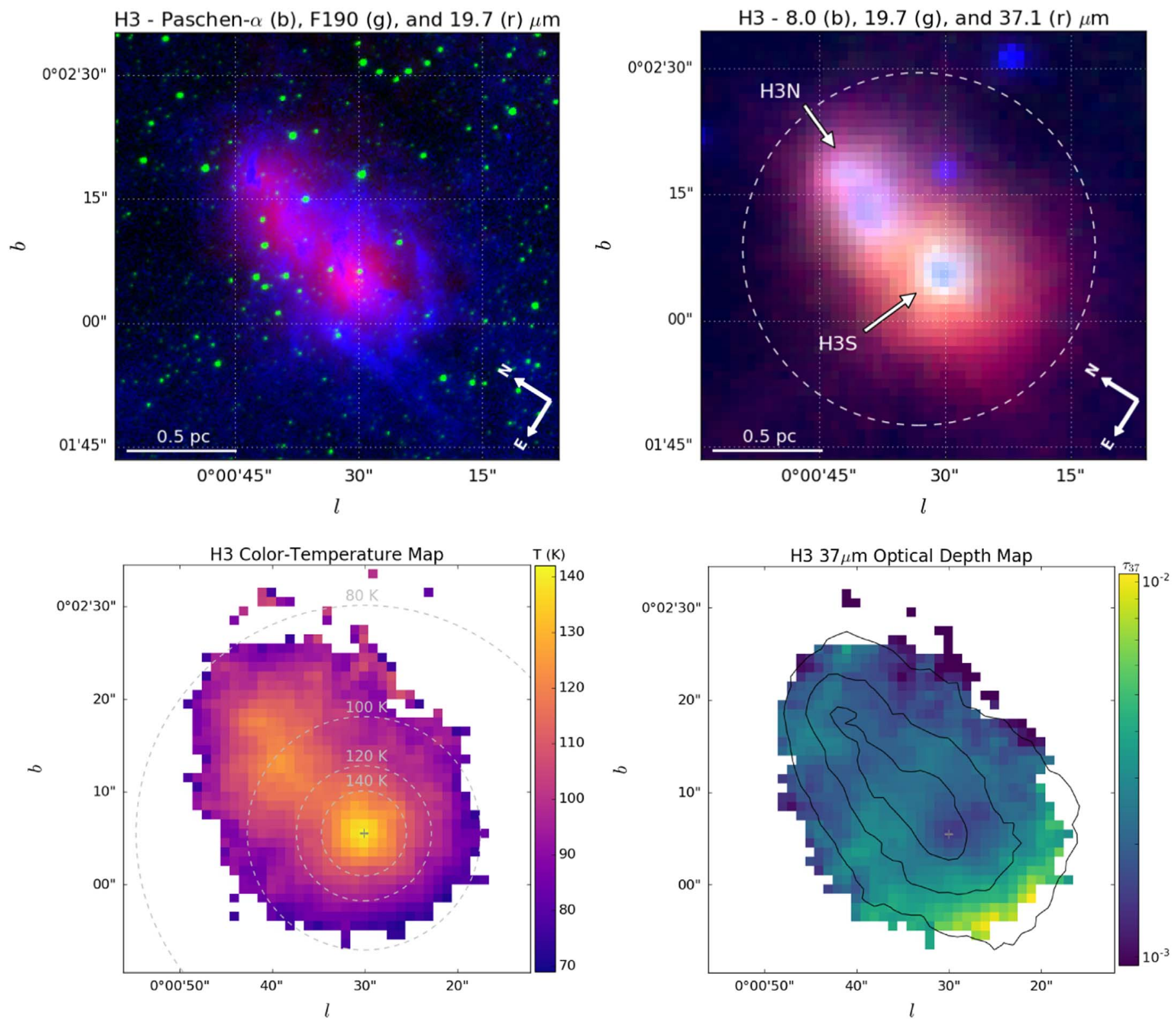

Figure 4. Top left: false-color image of $\mathrm{H} 3$ with HST Pa $\alpha$ (blue), HST F190 (green), and SOFIA/FORCAST $19.7 \mu \mathrm{m}$ (red). The ionizing source of H3 has not been identified in previous works, and its heating source is similarly unknown. Top right: false-color image of H3 with Spitzer/IRAC $8 \mu \mathrm{m}$ (blue), SOFIA/FORCAST $19.7 \mu \mathrm{m}$ (green), and SOFIA/FORCAST $37.1 \mu \mathrm{m}$ (red). The components of H3, south (H3S) and north (H3N), are indicated on the map. The photometric extraction area is shown by the dashed circle. Bottom left: color-temperature map of $\mathrm{H} 3$. Because a probable heating source of $\mathrm{H} 3$ is unknown, we assume that it is located at the position of the observed temperature maximum (indicated by the gray plus sign) and the radial temperature contours (gray dashed) have been positioned accordingly. Bottom right: $37 \mu \mathrm{m}$ optical depth map of $\mathrm{H} 3$ with $37 \mu \mathrm{m}$ contours (ranging from 0.3 to 0.9 in increments of $0.3 \mathrm{Jy}^{\text {pixel }}{ }^{-1}$ ).

\section{3. $\mathrm{H3}$}

$\mathrm{H} 3$ is the westernmost source in the $\mathrm{H}$ complex and is located at $l=00^{\circ} 00^{\prime} 32^{\prime \prime}, b=00^{\circ} 02^{\prime} 10^{\prime \prime}$ (Figure 1). Unlike H1 and $\mathrm{H} 2, \mathrm{H} 3$ does not have an identified candidate ionizing source. In Figure 4, we present multiwavelength imaging of $\mathrm{H} 3$, along with the color-temperature and optical depth maps of the source. The ionized gas emission associated with this $\mathrm{H}$ II region displays a filamentary structure and is slightly more extended than the warm dust emission observed by FORCAST. The surrounding environment of $\mathrm{H} 3$ appears to be less complex than $\mathrm{H} 1$ and $\mathrm{H} 2$, which have ionized gas extending well beyond the observed dust emission associated with the $\mathrm{HII}$ regions. In the mid-infrared, there are at least two spatially resolved emission peaks within the region that are separated by $\sim 0.5$ pc. The brightest source is located to the south $(\mathrm{H} 3 \mathrm{~S})$, with the others being present to the north $(\mathrm{H} 3 \mathrm{~N})$. In the Spitzer/IRAC maps of H3, there appear to be two $8 \mu \mathrm{m}$ sources coincident with the location of $\mathrm{H} 3 \mathrm{~N}$, which we refer to as $\mathrm{H} 3 \mathrm{~N} 1$ and $\mathrm{H} 3 \mathrm{~N} 2$.

The color-temperature map of $\mathrm{H} 3$ was produced using the 19.7 and $37.1 \mu \mathrm{m}$ FORCAST observations (Figure 4). The maximum temperature in the map $\left(T_{d}=140 \pm 21 \mathrm{~K}\right)$ occurs at the location of $\mathrm{H} 3 \mathrm{~S}$, and two distinct local maxima $\left(T_{d}=130 \pm 20 \mathrm{~K}\right.$ and $\left.T_{d}=120 \pm 18 \mathrm{~K}\right)$ are found in the northern portion of $\mathrm{H} 3$ corresponding to $\mathrm{H} 3 \mathrm{~N} 1$ and $\mathrm{H} 3 \mathrm{~N} 2$. Interpreting the detailed temperature structure of $\mathrm{H} 3$ is somewhat challenging because previous works have not identified a candidate stellar source associated with this object. In this analysis, we assume that the dust-heating source is close 

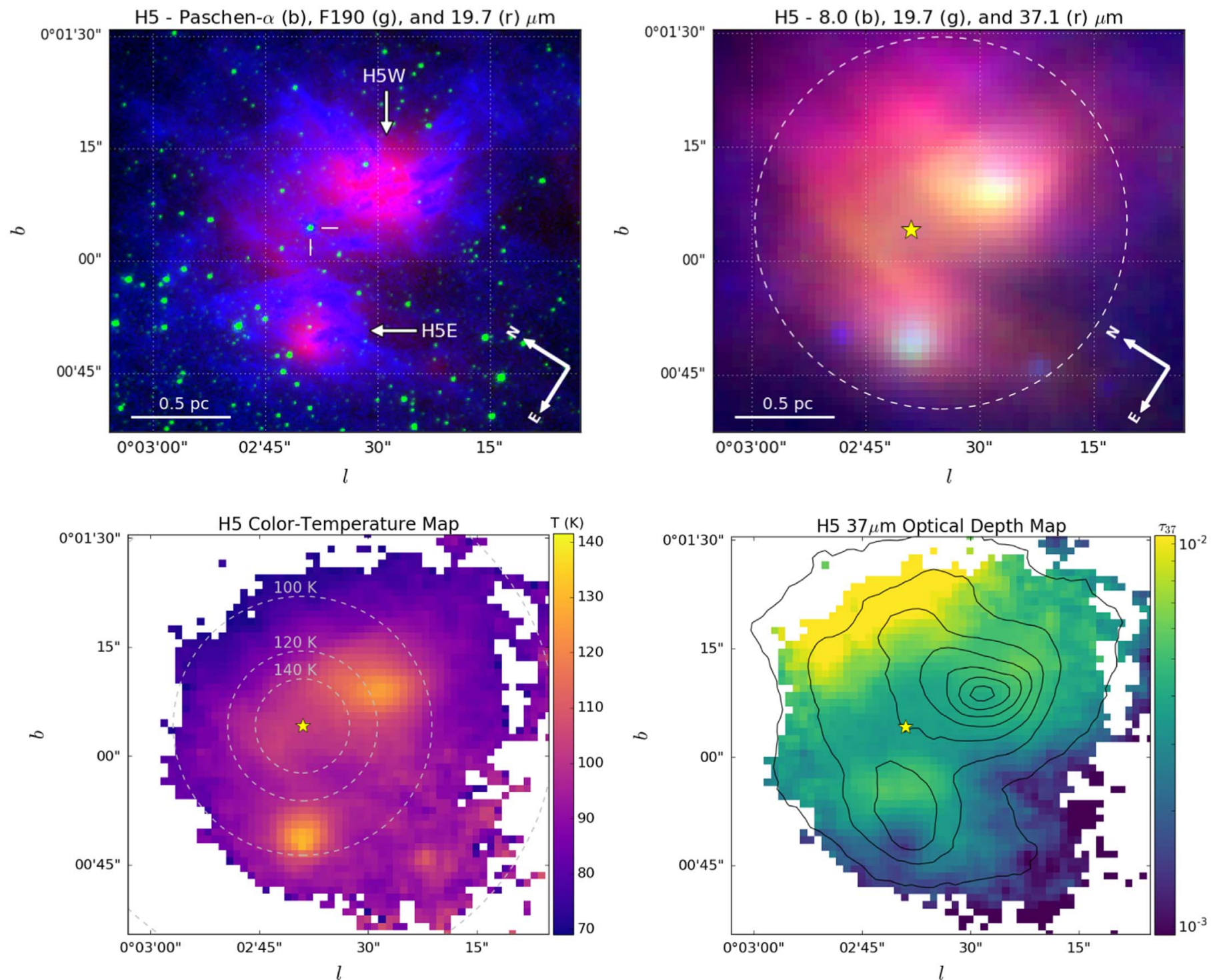

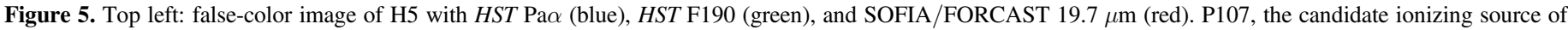

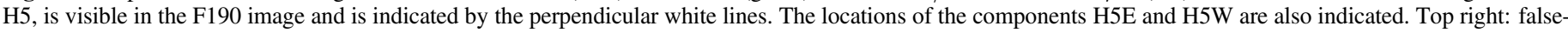

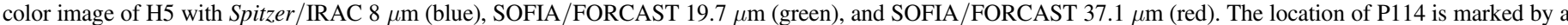

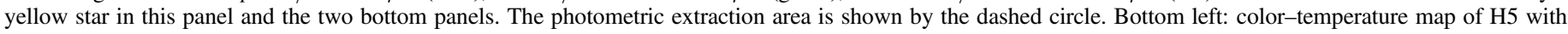

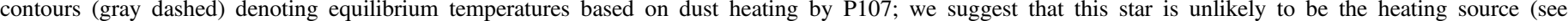
Section 4.4). Bottom right: $37 \mu \mathrm{m}$ optical depth map of $\mathrm{H} 5$ with $37 \mu \mathrm{m}$ contours (ranging from 0.2 to 1.4 in increments of $0.2 \mathrm{Jy}^{\text {pixel }}{ }^{-1}$ ).

to the maximum temperature peak. If so, the calculated temperature contours show that it cannot be responsible for heating dust in the northern part of $\mathrm{H} 3$ to the observed temperatures present in this region. This requires at least one additional heating source in the north, whose position could be indicated by the local temperature maxima in $\mathrm{H} 3 \mathrm{~N}$. We will return to this point in Section 4.6. The $37 \mu \mathrm{m}$ optical depth of $\mathrm{H} 3$ ranges between $4.5 \times 10^{-4}$ and $5.2 \times 10^{-3}$. The location of the temperature peak associated with $\mathrm{H} 3 \mathrm{~S}$ appears to correspond to a location of lesser dust column in the map; however, this may be an artifact of the line-of-sight temperature distribution, similar to what is observed in H2SE.

The SED model of $\mathrm{H} 3$ is best fit by a source having an infrared luminosity of $L_{\mathrm{IR}}=3.0_{-0.8}^{+0.5} \times 10^{5} L_{\odot}$ and dust mass $M_{d}=9.2_{-2.4}^{+1.4} \times 10^{-2} M_{\odot}$. As in the case of $\mathrm{H} 1$ and $\mathrm{H} 2$, the $\mathrm{PAH}$ abundance of the model was modified to improve the SED fit in the mid-infrared $\left(X_{\mathrm{PAH}}=0.3_{-0.25}^{+0.25}\right)$. Overall the dust model does a good job of reproducing the observed SED (reduced- $\chi^{2}=0.9$ ).

\section{4. $H 5$}

$\mathrm{H} 5$ is located in the northern half of the $\mathrm{H}$ complex at $l=00^{\circ} 02^{\prime} 34^{\prime \prime}, b=00^{\circ} 01^{\prime} 00^{\prime \prime}$ (Figure 1). Its ionizing source is thought to be the emission-line star P107, which is classified as a type O4-6If ${ }^{+}$(Dong et al. 2015). In Figure 5, we present multiwavelength imaging of H5 along with the colortemperature and optical depth maps of the source. The ionized gas emission in H5 shows a complex structure that is reminiscent of a bipolar morphology; however, this may only be an effect of the viewing geometry. The infrared dust emission appears to be concentrated into two primary sources, which we refer to as $\mathrm{H} 5 \mathrm{E}$ and $\mathrm{H} 5 \mathrm{~W}$. These components were originally identified in the radio by Zhao et al. (1993). 


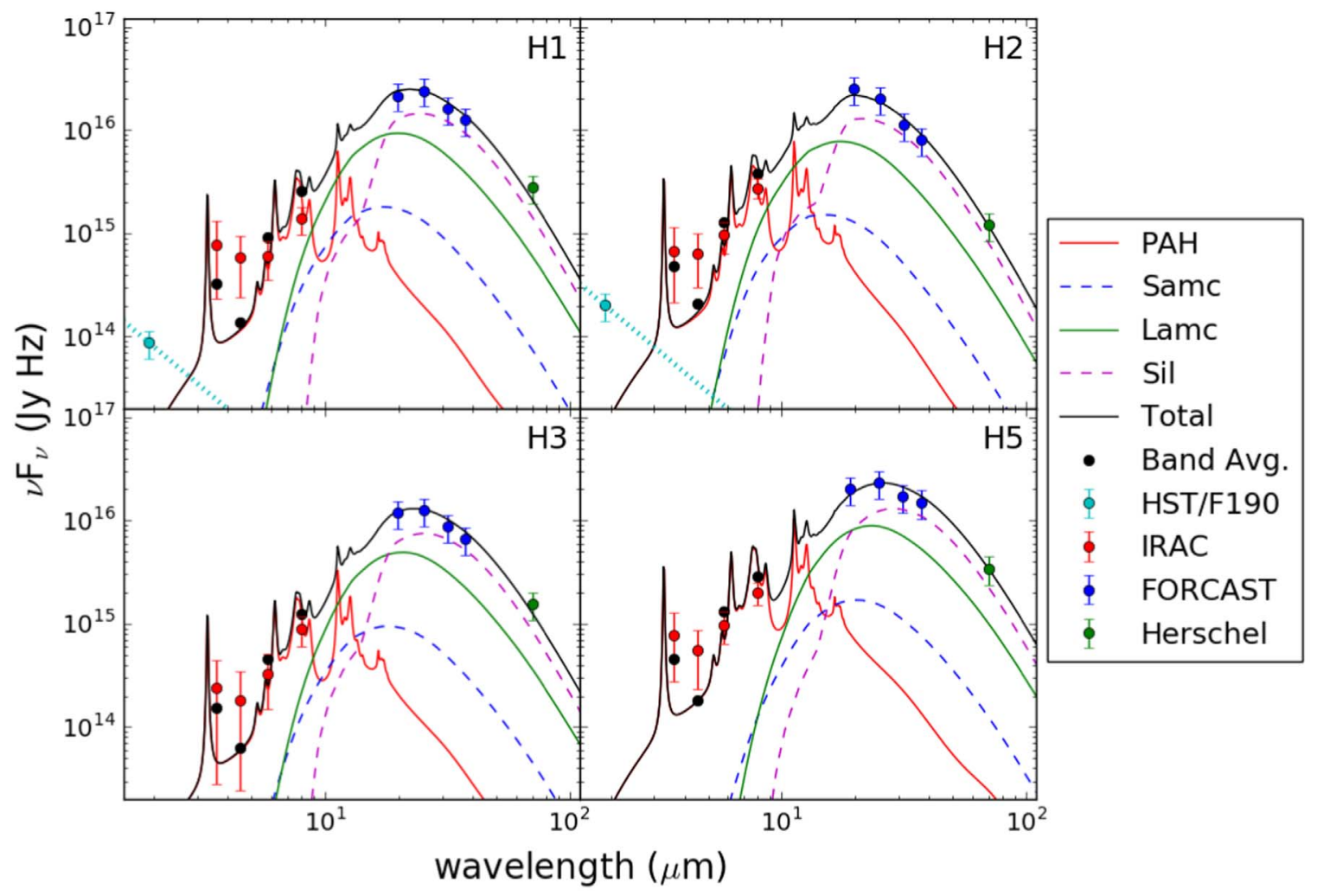

Figure 6. SED models of the H II regions H1, H2, H3, and H5. Details of the models are described in the text, and the best-fit parameters can be found in Table 3.

A color-temperature map of $\mathrm{H} 5$ was produced using the 19.7 and $37.1 \mu \mathrm{m}$ FORCAST data (Figure 5). There are two temperature peaks in the main portion of $\mathrm{H} 5$. These features correspond to the location of the bright infrared sources H5E and $\mathrm{H} 5 \mathrm{~W}$. Interestingly, the maps do not show a significant temperature increase at the location of P107, which implies that the observed dust at this location must be much farther from the star than it appears in projection. To examine the dust heating of the region in more detail, we show radial temperature contours at the location of P107 to determine whether it can reproduce the observed spatial distribution of temperatures. From this analysis, it is clear that the two temperature peaks are much warmer than one would expect if P107 was responsible for heating H5E and $\mathrm{H} 5 \mathrm{~W}$. This implies that local sources of heating are needed to explain the observed dust temperatures at these locations.

The fact that P107 does not appear to dominate the heating of the two bright infrared components in $\mathrm{H} 5$, or significantly warm dust in its (projected) surroundings, brings into question the association of this source with H5. Dong et al. (2015) have already noted a significant radial velocity offset between P107 and the ionized and molecular gas associated with $\mathrm{H} 5$ $\left(\Delta v \approx 60 \mathrm{~km} \mathrm{~s}^{-1}\right)$. Both of these pieces of evidence suggest that P107 is actually external to the $\mathrm{H}$ II region. Given the high density of sources in this region of the GC, P107 could simply be a chance alignment with $\mathrm{H} 5$. However, if P107 is within $2.5 \mathrm{pc}$, it could still be responsible for ionizing the gas in $\mathrm{H} 5 .{ }^{13}$ Such a scenario is not inconsistent with the observed dust temperatures in $\mathrm{H} 5$, though local sources of dust heating would still be required for H5E and H5W. Alternatively, H5 could be

\footnotetext{
13 This is based on calculating the maximum distance at which P107 could supply a sufficient number of ionizing photons after accounting for the geometric dilution factor. We have assumed that P107 is an O4I star and used the properties of H5 measured by Zhao et al. (1993).
}

ionized by star(s) local to the $\mathrm{H}$ II region. Radio continuum observations of $\mathrm{H} 5$ suggest that the ionization of $\mathrm{H} 5 \mathrm{~W}$ and $\mathrm{H} 5 \mathrm{E}$ could be accomplished by an $\mathrm{O} 6.5 \mathrm{~V}$ and $\mathrm{O} 8 \mathrm{~V}$ star, respectively (Zhao et al. 1993). However, no massive stars have previously been identified in these regions. We will return to this analysis and examine possible heating sources for $\mathrm{H} 5 \mathrm{E}$ and $\mathrm{H} 5 \mathrm{~W}$ in Section 4.6.3.

The $37 \mu \mathrm{m}$ optical depth of $\mathrm{H} 5$ ranges between $9.8 \times 10^{-4}$ and $1.1 \times 10^{-2}$. The column density near the peak location of $\mathrm{H} 5 \mathrm{~W}$ is fairly uniform, while there are more prominent variations near H5E. In the northern part of H5, the column density increases to $\tau_{37} \approx 10^{-2}$, which is consistent with the location of relatively cool dust temperatures of $\sim 75 \mathrm{~K}$ and increased $70 \mu \mathrm{m}$ emission. The column density does not show any significant variations near the location of P107, which is consistent with the dust being relatively unaffected by the star.

The SED model of H5 is best fit by a source having an infrared luminosity of $L_{\mathrm{IR}}=5.7_{-1.5}^{+0.8} \times 10^{5} L_{\odot}$ and dust mass of $M_{d}=3.4_{-0.9}^{+0.5} \times 10^{-1} M_{\odot}$. The PAH abundance of the model is best fit by $X_{\mathrm{PAH}}=0.5_{-0.25}^{+0.25}$. The model results discussed in this section and presented in Figure 6 consider emission from the entirety of $\mathrm{H} 5$ rather than dividing it into separate components. The sources $\mathrm{H} 5 \mathrm{E}$ and $\mathrm{H} 5 \mathrm{~W}$ are discussed further in Section 4.6.3.

\subsection{Faint or Compact H II Regions}

Several additional $\mathrm{H}$ II regions were also detected in the FORCAST data, including H4, H6, H7, H10, H11, H12, and $\mathrm{H} 14^{14}$ (Figure 1). Analysis of these sources, excluding H12,

\footnotetext{
${ }^{14}$ The $\mathrm{H}$ II region that we have designated as H14 was incorrectly labeled as H12 by Dong et al. (2015) in their Figure 1. H12 was originally reported by Lang et al. (2001) and is located to the Galactic east of H14 (see our Figure 1).
} 


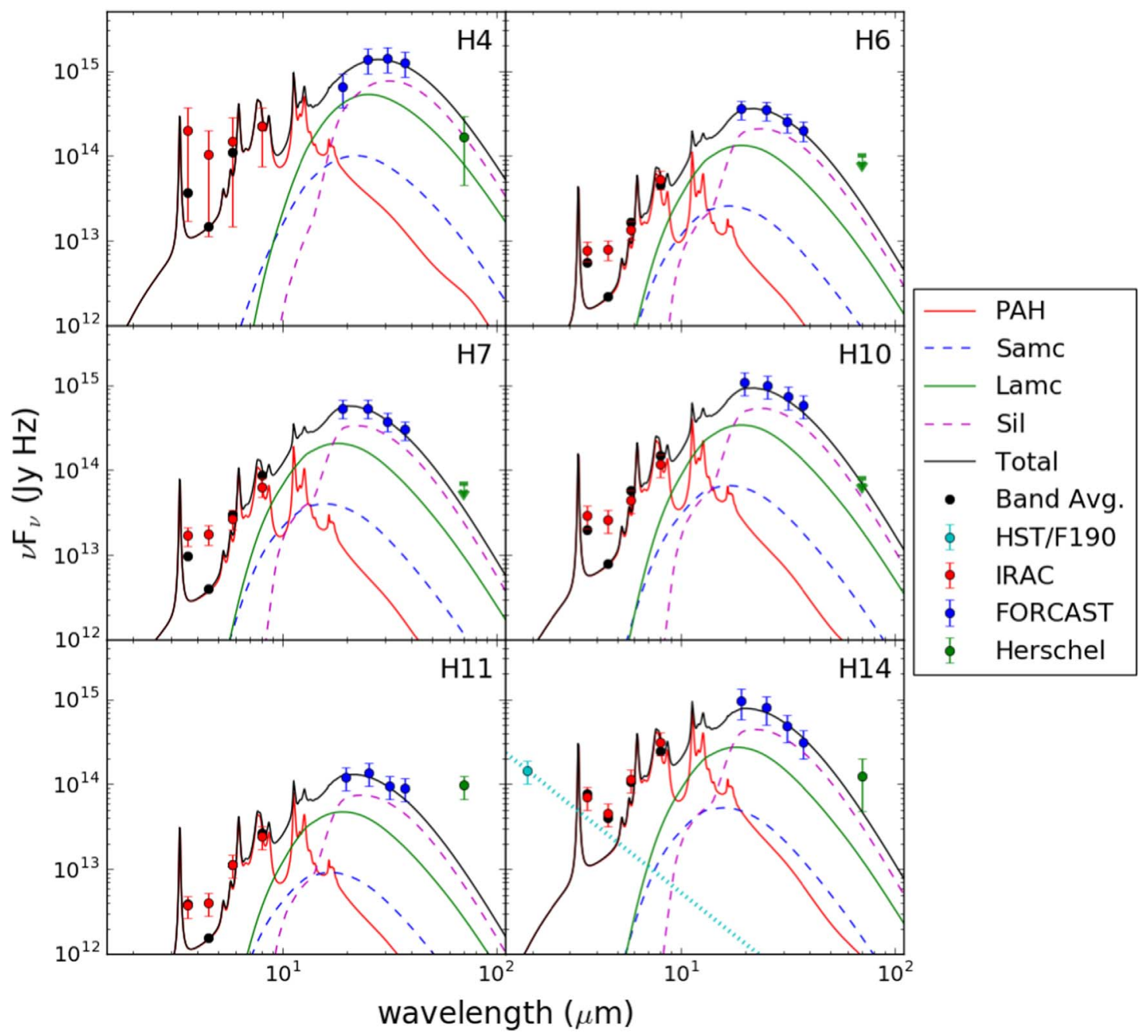

Figure 7. SED models for the remaining H II regions: H4, H6, H7, H10, H11, and H14. Details of the models are described in the text, and the best-fit parameters can be found in Table 3.

which is detected at low significance, ${ }^{15}$ are presented together in this section because they are more compact than $\mathrm{H} 1, \mathrm{H} 2, \mathrm{H} 3$, and H5. The sources $\mathrm{H} 6$ and $\mathrm{H} 11$ are consistent with the size of the FORCAST beam at $19.7 \mu \mathrm{m}\left(\mathrm{FWHM}_{19.7}=3\right.$ !" 2$)$. $\mathrm{H} 7$ and $\mathrm{H} 10$ are only marginally resolved with $\mathrm{FWHM}_{19.7}=4^{\prime \prime} \times 3$ ". 8 and $\mathrm{FWHM}_{19.7}=4$ !" $8 \times 3$ "!9, respectively. $\mathrm{H} 4$ and $\mathrm{H} 14$ are both spatially extended, though these objects are detected at modest significance $(\sim 3 \sigma$ per pixel at $19.7 \mu \mathrm{m})$. To study these objects, we produce SED models using DustEM following the process used for the other H II regions. These models are displayed in Figure 7.

$\mathrm{H} 4$ is best fit by a source with an infrared luminosity of $L_{\mathrm{IR}}=3.5_{-1.0}^{+0.7} \times 10^{4} L_{\odot}$. The adopted dust model does a relatively good job of reproducing the observed SED, and we find that the PAH abundance is consistent with the standard value from Compiègne et al. (2011). H6 and $\mathrm{H} 7$ are best fit by sources with infrared luminosities of $L_{\mathrm{IR}}=8.3_{-1.5}^{+1.8} \times 10^{3} L_{\odot}$ and $L_{\mathrm{IR}}=1.3_{-0.3}^{+0.3} \times 10^{4} L_{\odot}$, respectively. These objects show a slight depletion in PAHs compared to H4. Both models have difficulty reproducing the observed fluxes in the IRAC bands (in particular channel 2 , which shows a $\gtrsim 4 \sigma$ excess over the

\footnotetext{
15 Of the remaining known sources in the $\mathrm{H}$ complex, only $\mathrm{H} 9$ and H13 are clearly nondetections. H8 lies outside of the observed fields.
}

model in both sources, as well as H10). This discrepancy at the shortest wavelengths likely comes from the contribution of the stellar sources associated with $\mathrm{H} 6, \mathrm{H} 7$, and H10. ${ }^{16}$ For example, the model of $\mathrm{H} 14$, which has a known stellar counterpart, would miss badly on the shortest IRAC bands if not for the contribution of its star.

$\mathrm{H} 10$ and $\mathrm{H} 11$ are best fit by sources with infrared luminosities of $L_{\mathrm{IR}}=2.2_{-0.6}^{+0.6} \times 10^{4} L_{\odot}$ and $L_{\mathrm{IR}}=3.2_{-1.0}^{+1.1} \times 10^{3} L_{\odot}$, respectively. The PAH abundance of $\mathrm{H} 11$ is consistent with the value of Compiègne et al. (2011), while $\mathrm{H} 10$ may have a slight depletion in this component $\left(X_{\mathrm{PAH}}=0.7_{-0.30}^{+0.25}\right)$. Similar to $\mathrm{H6}$ and $\mathrm{H} 7$, both models have difficulty reproducing the observed fluxes in the shortest-wavelength IRAC bands. Other than this, the model does a relatively good job of reproducing the observed emission in H10. In contrast, the dust emission from H11 is well fit at FORCAST wavelengths but shows a large far-infrared excess that cannot be reproduced by the model. H11 is the lone source that shows a significant excess at $70 \mu \mathrm{m}$. This emission is likely related to a cold dust component that may be associated with $\mathrm{H} 11$ or a spatially coincident background source. Our data cannot differentiate these scenarios. If the far-infrared emission is associated with $\mathrm{H} 11$, the

\footnotetext{
${ }^{16}$ It is highly unlikely that the stellar contamination is caused by an unassociated source because $\mathrm{H} 6$ and $\mathrm{H} 7$ are both unresolved by FORCAST while $\mathrm{H} 10$ is only marginally resolved.
} 
Table 4

Summary of Heating Source Properties

\begin{tabular}{|c|c|c|c|c|c|}
\hline Region & $T_{\text {peak }}(\mathrm{K})$ & Location $(l, b)^{\mathrm{a}}$ & $d\left(^{\prime \prime \prime}\right)^{\mathrm{b}}$ & $N_{\text {source }} \mathrm{c}$ & Candidate Source \\
\hline H1 & $130 \pm 20$ & $(359.9912,0.0132)$ & $7.6_{-3.3}^{+5.9}$ & 238 & P114 \\
\hline $\mathrm{H} 2 \mathrm{NE}$ & $210 \pm 32$ & $(359.9848,0.0271)$ & $1.8_{-0.8}^{+1.3}$ & 10 & P35 \\
\hline $\mathrm{H} 3 \mathrm{~S}$ & $140 \pm 21$ & $(0.0083,0.0345)$ & $2.6_{-1.6}^{+1.9}$ & 32 & 2MASS J17453016-2854387 \\
\hline $\mathrm{H} 3 \mathrm{~N} 1$ & $130 \pm 20$ & $(0.0109,0.0371)$ & $3.3_{-1.1}^{+2.0}$ & 33 & 2MASS J17452987-2854289 \\
\hline $\mathrm{H} 3 \mathrm{~N} 2$ & $120 \pm 18$ & $(0.0114,0.0382)$ & $4.2_{-1.4}^{+2.7 \mathrm{e}}$ & 47 & 2MASS J17452987-2854289 \\
\hline
\end{tabular}

Notes.

a The location of the observed 19/37 color-temperature maximum in Galactic coordinates. Typical errors in the position are $\lesssim 1$ "! 5 .

${ }^{\mathrm{b}}$ Equilibrium heating distance determined from Equation (1), where $L_{\text {source }} \approx L_{\mathrm{IR}}$ has been adopted.

c The number of near-infrared continuum sources listed in the HST Pa $\alpha$ catalog within $d$.

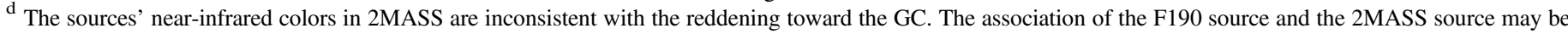
problematic. See the discussion in Section 4.6.

${ }^{\mathrm{e}} d$ for $\mathrm{H} 3 \mathrm{~N}$ was calculated assuming a source luminosity $L=10^{5} L_{\odot}$.

total infrared luminosity of the source would be boosted to $L_{\mathrm{IR}} \approx 4.6 \times 10^{3} L_{\odot}$, which is just outside the fitted SED model $\left(L_{\mathrm{IR}}=3.2_{-1.0}^{+1.1} \times 10^{3} L_{\odot}\right)$.

H14 is best fit by a source having an infrared luminosity of $L_{\mathrm{IR}}=2.0_{-0.4}^{+0.5} \times 10^{4} L_{\odot}$ and the relative PAH abundance that is larger than that of Compiègne et al. (2011). Unlike the other sources discussed in this section, $\mathrm{H} 14$ has an identified candidate ionizing source. $\mathrm{P} 100$ is classified as a type O4-6If ${ }^{+}$(Dong et al. 2015), which is similar in subtype to P114. Even though their stars are similar, H14 is quite different from $\mathrm{H} 1$. $\mathrm{H} 1 \mathrm{has}$ an observed infrared luminosity comparable to the stellar luminosity of P114 (i.e., $L_{\mathrm{IR}} / L_{\star} \sim 1$ ), while H14 and P100 have $L_{\mathrm{IR}} / L_{\star} \sim 0.1$. This implies that a large fraction of the photons are escaping the local H II region rather than being absorbed by dust and reradiated in the infrared. H14 is the only source in this study that shows evidence for a large escape fraction of photons. While this could be true of other objects in the region, we lack constraints on stellar sources, which are needed to investigate this further.

\subsection{Identifying Candidate Heating Sources}

In this section, we attempt to identify candidate heating sources associated with $\mathrm{H} 2, \mathrm{H} 3$, and H5. First, we use colortemperature maps of the $\mathrm{H}$ II regions to constrain the location of possible heating sources. Next, we search near-infrared source catalogs to find stars coincident with these locations that might be associated with the H II regions. We use both the HST Pa $\alpha$ survey of the GC (Dong et al. 2011) and 2MASS (Cutri et al. 2003) catalogs to examine potential stellar sources. While the $H S T$ data offer superior angular resolution $(\sim 0$." 2$)$ compared to 2MASS $\left(\sim 2^{\prime \prime}\right)$, the $\mathrm{Pa} \alpha$ survey only provides a single continuum filter. In contrast, 2 MASS provides multiple nearinfrared colors $\left(J, H\right.$, and $\left.K_{s}\right)$, which are useful for determining whether the source is consistent with the reddening toward the GC. However, there are significant limitations with the 2MASS data because of source confusion in crowded fields. In addition to near-infrared sources, We also provide mid-infrared counterparts for the FORCAST sources from the Spitzer IRAC survey of the GC (Ramírez et al. 2008). Discussion pertaining to H2, $\mathrm{H} 3$, and $\mathrm{H} 5$ can be found in the following subsections, and a summary of the results can be found in Table 4 .

\subsection{1. $\mathrm{H} 2$}

Examining the morphology of $\mathrm{H} 2$ reveals two distinct components: H2NE and H2SW (Figure 3). Our analysis shows that $\mathrm{H} 2 \mathrm{NE}$ is consistent with being heated by its candidate ionizing source, P35; however, H2SW appears to be heated independently. To study H2SW in more detail, we estimate its luminosity by fitting an emissivity-modified blackbody to the SED. We extract photometry of the source ${ }^{17}$ and use the observed 19/37 color temperature for the fit. This results in an infrared luminosity of $8.7_{-2.0}^{+1.8} \times 10^{3} L_{\odot}$ and is consistent with a B1.5V star (Pecaut \& Mamajek 2013). If the central source in $\mathrm{H} 2 \mathrm{SW}$ is a B-type star, it may explain the lack of ionized gas emission associated with this source. The discovery of H2SW demonstrates that the combination of SOFIA/FORCAST sensitivity and spatial resolution is capable of identifying relatively luminous $\left(\sim 10^{4} L_{\odot}\right)$ stars that may not produce ionizing radiation.

To search for a near-infrared counterpart to H2SW, we use Equation (1) to estimate the maximum distance at which the heating source can be present from the observed temperature peak $\left(T_{d}=110 \pm 17 \mathrm{~K}\right)$. If the infrared luminosity of $\mathrm{H} 2 \mathrm{SW}$ is the same as the stellar luminosity of its heating source $\left(L_{\star} \approx L_{\mathrm{IR}}\right)$, it would require the source to be within $1.3_{-0.4}^{+1.6}$ arcsec of the observed temperature peak. We identify nearinfrared sources in this region using the continuum source catalog from the HST GC $\mathrm{Pa} \alpha$ survey (Dong et al. 2011). A total of nine sources are contained within the search area for $\mathrm{H} 2 \mathrm{SW}$, and none are candidate $\mathrm{Pa} \alpha$ emission-line sources. Only one source has a cross-matched 2MASS catalog entry (2MASS J17452794-2856230), and it is identified as the counterpart to the sole IRAC source in the search radius (SSTGC 0492222). Interestingly, an earlier study of infrared sources in the GC by An et al. (2011) has ruled out this IRAC source as a massive YSO based on its mid-infrared spectra.

Sources consistent with being at the distance of the GC are highly reddened because of interstellar extinction. 2MASS $\mathrm{J} 17452794-2856230$ is not detected in either 2MASS $J$ or $H$

\footnotetext{
17 For other sources, we determine the aperture based on the $37 \mu \mathrm{m}$ size; however, the extended emission in the surrounding region of $\mathrm{H} 2 \mathrm{SW}$ dominates the point source at this wavelength. Instead, we use an aperture consistent with its $25 \mu \mathrm{m}$ size $(r=4 ! .2)$.
} 
band; however, based on the $H$-band upper limit, the color of the source is $H-K_{s}>1.4$. Prior work on near-infrared sources in the H complex by Dong et al. (2015) indicates that sources in this region have a range of $H-K$ values from 1.3 to 2.4 with a mean value of 1.7. A more recent study of near-infrared sources over a larger portion of the GC by Nogueras-Lara et al. (2018) finds a mean $H-K_{s}$ value of $\sim 2$; however, they also find evidence for a low-extinction population with $H-K_{s} \sim 1.5$. Considering the limited near-infrared color information for this source, it is difficult to positively place it at the GC, but it remains a promising potential counterpart to $\mathrm{H} 2 \mathrm{SW}$.

\subsection{2. $H 3$}

Currently, the identities of the ionization and heating source (s) of H3 are unknown. Based on the total Lyman continuum flux of $\mathrm{H} 3$, the ionization source is consistent with an $\mathrm{O} 7.5 \mathrm{~V}$ star (Zhao et al. 1993). Our analysis of the color-temperature map of $\mathrm{H} 3$ indicates three distinct temperature peaks in the region, which matches the number of observed $8 \mu \mathrm{m}$ sources. The maximum dust temperature in the region occurs at the location of $\mathrm{H} 3 \mathrm{~S}\left(T_{d}=140 \pm 21 \mathrm{~K}\right)$. To study H3S in more detail, we extracted photometry using an aperture centered on the source with radius $r=8^{\prime \prime}$, which is sufficiently large to capture the extended emission in the southernmost part of $\mathrm{H} 3$. The source luminosity was determined by using the observed 19/37 color temperature and fitting an emissivity-modified blackbody to the SED. This results in an infrared luminosity of $9.5_{-2.0}^{+1.5} \times 10^{4} L_{\odot}$, which is consistent with an $07.5 \mathrm{~V}$ star. Similar to our analysis of $\mathrm{H} 2 \mathrm{SW}$, we estimate the maximum distance at which the heating source can be present in order to produce the observed temperature maximum. If the infrared luminosity of $\mathrm{H} 3 \mathrm{~S}$ is the same as the luminosity of the heating source (i.e., $L_{\star} \approx L_{\mathrm{IR}}$ ), it would require the star to be within $2.6_{-1.6}^{+1.9} \operatorname{arcsec}$ of the observed temperature peak.

A search of the HST GC catalog results in 32 near-infrared continuum sources that are contained to the relevant search area. As in the case of $\mathrm{H} 2 \mathrm{SW}$, there is limited near-infrared color information for the majority of these sources (only two 2MASS catalog entries). There is one identified IRAC source inside the search radius, SSTGC 0498297, which appears to be the mid-infrared counterpart to H3S. The IRAC GC catalog matches this source with the near-infrared counterpart 2MASS $\mathrm{J} 17453016-2854387$. The 2MASS source is detected at $J$ band but not $H$ or $K$ band. Given this information, it seems unlikely that the 2MASS source is consistent with the reddening toward the GC. Because of the high number density of sources in the region, 2MASS has significant issues with confusion. In this case, the near-infrared source association is likely problematic.

Potential heating sources were also examined in the northern part of H3. There are two local temperature maxima in the region that are separated by only $2^{\prime \prime}$. Given that their projected distance is relatively small and their peak temperatures are comparable $(130 \pm 20 \mathrm{~K}$ and $120 \pm 18 \mathrm{~K})$, both maxima could be produced by a single source. If the source is similar in luminosity to $\mathrm{H} 3 \mathrm{~S}\left(L_{\star} \approx L_{\mathrm{IR}} \approx 1 \times 10^{5} L_{\odot}\right)$, its proximity to the observed temperature peaks can be estimated using Equation (1). For the 130 and $120 \mathrm{~K}$ temperature peaks, the heating source should be within $3.3_{-1.1}^{+2.1}$ and $4.2_{-1.4}^{+2.7}$ arcsec of these locations.

A search of the HST GC catalog for sources common to both regions results in 17 objects. Interestingly, one of the sources is identified as a candidate $\operatorname{Pa} \alpha$ emission-line source by
Dong et al. (2011) (secondary candidate source ID 152). Because emission-line stars are typically luminous O-type or Wolf-Rayet stars (Mauerhan et al. 2010), this source is a promising candidate to ionize and heat dust in the region, though spectral classification is needed to confirm its exact stellar type to better assess this scenario. We cross-match the $\operatorname{Pa} \alpha$ source location with the 2MASS catalog and find a possible counterpart (2MASS J17452987-2854289). The nearinfrared colors of the 2MASS source $\left(H-K_{s}=1.5\right)$ are consistent with the value of other stars in the region studied by Dong et al. (2015), which makes this a promising association.

\subsection{3. $H 5$}

As noted in our prior discussion of $\mathrm{H} 5$, the ionization and heating sources may not be the same for this $\mathrm{H}$ II region, which makes it one of the more complex regions studied in this work. Analysis of the distribution of dust temperatures clearly shows that P107 cannot be responsible for creating the observed temperature peaks (Figure 5). Instead, local heating sources are required for each of these regions. We extract photometry for both infrared sources (H5E and H5W) using an aperture centered on the source with radius $r=10^{\prime \prime}$. The luminosities of the sources are estimated using the observed 19/37 color temperatures and fitting an emissivity-modified blackbody to the source SEDs. This process results in infrared luminosities of $1.7_{-0.5}^{+0.5} \times 10^{5} L_{\odot}$ for $\mathrm{H} 5 \mathrm{~W}$ and $8.8_{-2.6}^{+2.2} \times 10^{4} L_{\odot}$ for H5E.

Using Equation (1), we determine that the heating sources in $\mathrm{H} 5$ need to be within a radius of $4.9_{-2.2}^{+4.1}$ arcsec for $\mathrm{H} 5 \mathrm{~W}$ and $3.1_{-1.4}^{+2.6}$ arcsec for H5E. Searching the HST GC catalog in the relevant areas returns 75 near-infrared continuum sources for H5W and 27 sources for H5E. The vast majority of the [F190] sources lack 2MASS counterparts, which makes it difficult to assess their near-infrared color and possible association with the $\mathrm{H}$ II region. The apparent mid-infrared counterparts to $\mathrm{H} 5 \mathrm{E}$ and H5W (SSTGC 0525666 and SSTGC 0520760) have crossmatched 2MASS sources in the IRAC GC catalog (2MASS J17453839-2853265 and 2MASS J17454020-2853280). However, neither 2MASS source is detected in $J$ or $H$ band, which makes their association difficult to assess based on this information.

Returning to the F190 data, we note two peculiar sources with extended arc-like circumstellar emission (Figure 8). The near-infrared arcs could be indicative of reflection nebulae associated with the sources or very hot dust emission. Of the $\mathrm{H}$ II regions studied in this work, only $\mathrm{H} 1, \mathrm{H} 2$, and $\mathrm{H} 10$ display a similar extended morphology in the [F190] maps. The spatial coincidence of the mid-infrared sources (H5E and H5W) and the near-infrared nebulae is suggestive that they are associated; however, further study is needed to confirm this hypothesis. Spectral classification of the near-infrared sources is needed to examine whether they are sufficiently luminous to power the observed dust emission in the region.

\section{Discussion}

\subsection{Origin of the HII Regions}

There are numerous massive field stars in the GC whose origins are uncertain (e.g., Mauerhan et al. 2010), including the sources associated with the $\mathrm{H} \mathrm{H}$ II regions. A promising theory to explain the observed spatial distribution of the field stars is that they are former members of clusters that have been 

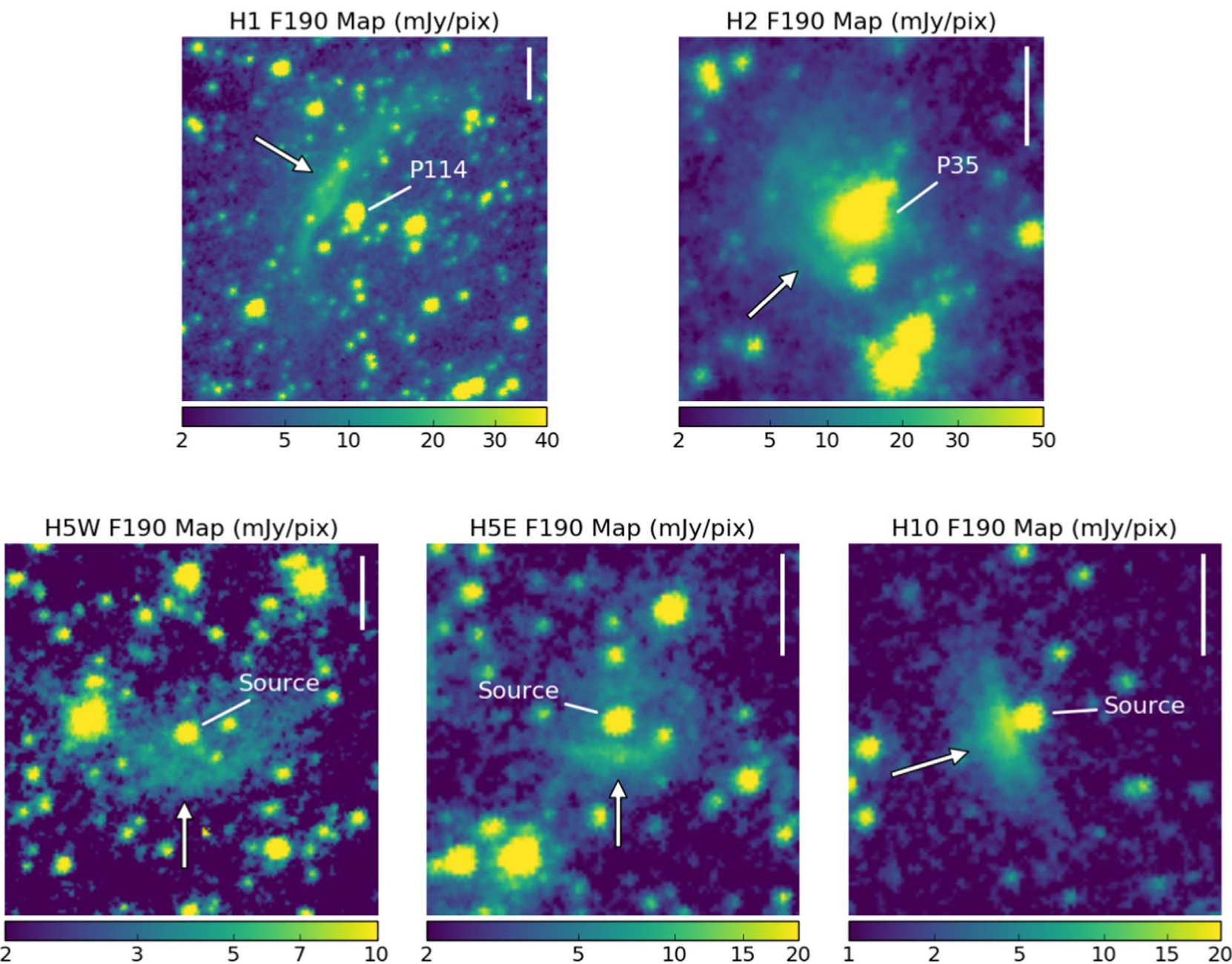

Figure 8. Cutouts of the HST F190 continuum observations for H1, H2, H5W, H5E, and H10. Each of these sources shows extended emission, which is likely generated from very hot dust $(\sim 1200 \mathrm{~K})$ or a reflection nebula. The arrows in the cutouts point to extended emission, and the near-infrared continuum sources that appear to be associated with these structures are also indicated. The extended emission associated with H1, H5E, and H5W has clear arc-like morphologies. A scale bar of $0.1 \mathrm{pc}$ is indicated in the upper right corner of each of the panels.

either dynamically ejected or stripped away via tidal forces (Habibi et al. 2014). For the stars in and near the H complex, there is a reasonable argument that many of them are part of the tidal tail of the Arches cluster given their respective positions and the expected dispersion of sources along galactic longitude due to tidal forces acting on the cluster (see Figure 3 of Habibi et al. 2014). However, there are reasons to doubt this hypothesis for all stars associated with the $\mathrm{H}$ complex. In particular, Dong et al. (2015) show that the velocity of the ionized gas near P35 is consistent with being driven by a pressure gradient caused by the ionization of the star (commonly referred to as the "champagne flow"), suggesting that it formed in situ.

Examining warm dust emission associated with the H II regions can be used to provide evidence for or against in situ formation for their associated stars. In spatially resolved objects, we consider the morphology of the $\mathrm{H}$ II region as evidence of the origin of the associated stellar source. We also measure the observed $19.7 \mu \mathrm{m}$ emission as a proxy for the $\mathrm{H}$ II region size and compare these values with models of $\mathrm{H}$ II regions with different physical conditions. We calculate both the Strömgren radius for an idealized stationary H II region (Strömgren 1939) and the size of a bow shock produced by a star traversing through the ISM (following from Wilkin 1996). The Strömgren radius, $R_{\text {Strömgren }}$, is given by

$$
R_{\text {Strömgren }}=\left(\frac{3 N_{\mathrm{LyC}}}{4 \pi \alpha_{\mathrm{H}} n_{e}^{2}}\right)^{1 / 3},
$$

where $N_{\mathrm{LyC}}$ is the number of ionizing photons produced by the star, $\alpha_{\mathrm{H}}$ is the hydrogen recombination rate $\left(\sim 3 \times 10^{-13} \mathrm{~cm}^{3} \mathrm{~s}^{-1}\right.$; Draine 2011), and $n_{e}$ is the electron number density. Physically, $R_{\text {Strömgren }}$ represents the radius at which all ionizing photons produced by a star are absorbed by the surrounding ISM. It assumes that the H II region is static and sufficient time has passed for the $\mathrm{H}$ II region to reach an equilibrium configuration. In contrast, a star traveling through the ISM will also act to shape its surroundings via its relative motion and winds. Momentum balance between the star's winds and intervening material is achieved at the standoff radius, $R_{\text {standoff }}$, which is given by

$$
R_{\text {standoff }}=\left(\frac{\dot{M}_{w} v_{w}}{4 \pi \rho v_{\star}^{2}}\right)^{1 / 2}
$$



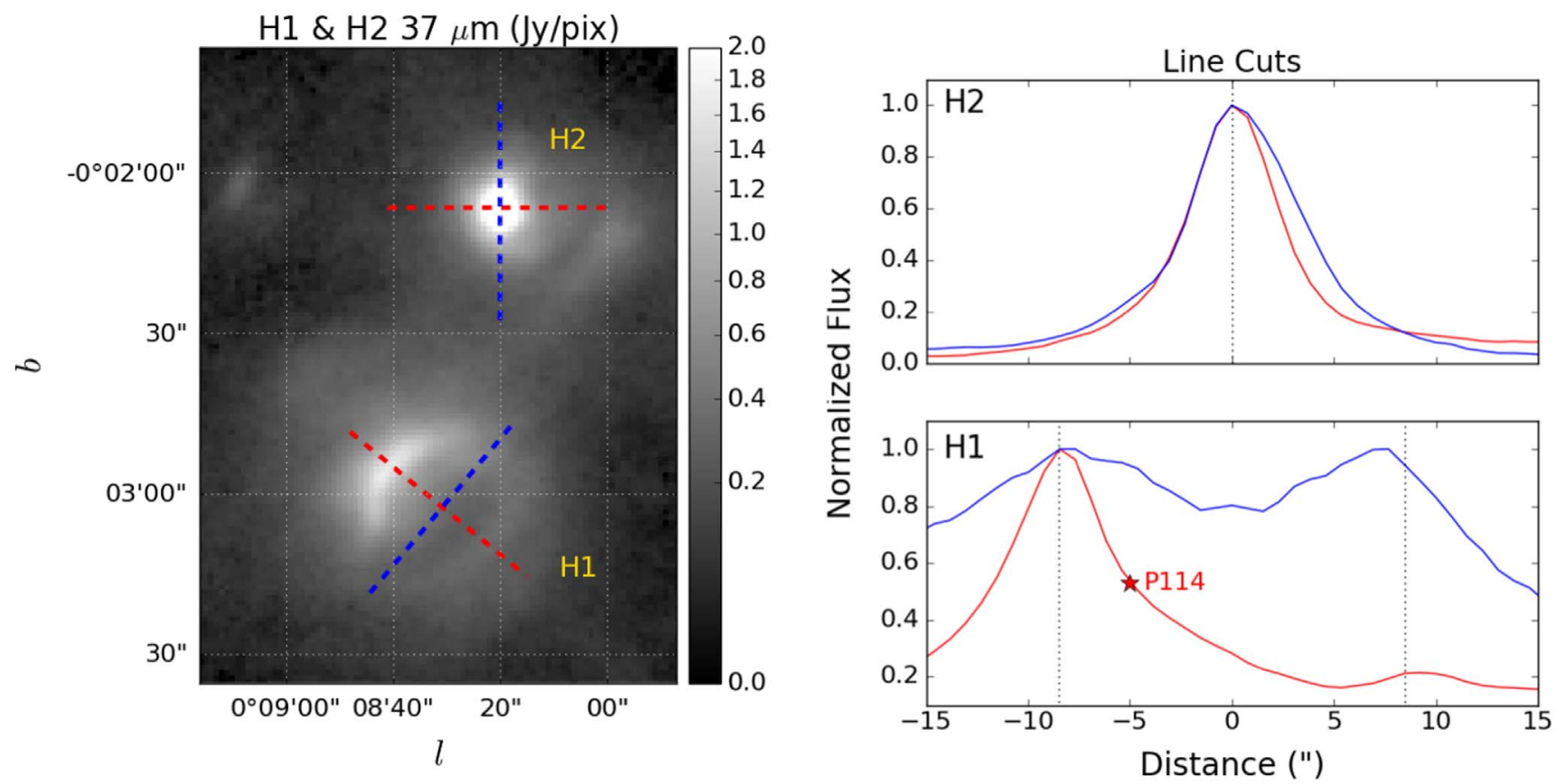

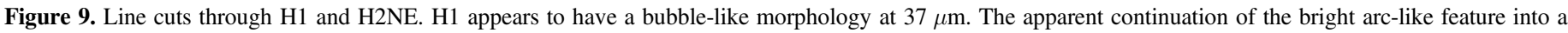

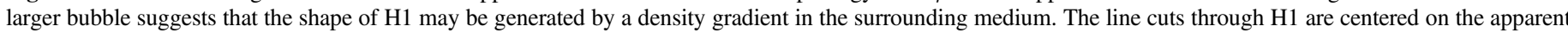

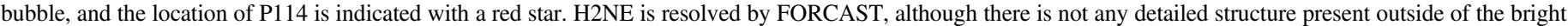
central peak, which corresponds to the location of P35.

where $\dot{M}_{w}$ and $v_{w}$ are the mass-loss rate and velocity of the stellar wind, respectively, $v_{\star}$ is the velocity of the star through the ambient medium, and $\rho$ is the density of the medium. For these calculations, we adopt $\dot{M}_{w} \approx 4 \times 10^{-6} M_{\odot} \mathrm{yr}^{-1}$ and $v_{w} \approx$ $2300 \mathrm{~km} \mathrm{~s}^{-1}$, which are typical of O I stars (Lamers \& Leitherer 1993).

FORCAST observations of H2NE show a somewhat compact morphology (Figure 9). Its deconvolved size at $19.7 \mu \mathrm{m}$ corresponds to $0.26 \pm 0.07 \mathrm{pc} \times 0.21 \pm 0.07 \mathrm{pc}$. Zhao et al. (1993) measured properties of H2, including the electron density $\left(n_{e}=3 \times 10^{3} \mathrm{~cm}^{-3}\right)$ and number of ionizing photons $\left(\log \left(N_{\mathrm{LyC}}\left[\mathrm{s}^{-1}\right]\right)=48.6\right)$. Based on these parameters, we find $R_{\text {Strömgren }}=0.2 \mathrm{pc}$. The agreement between $R_{\text {Strömgren }}$ and the measured size of the source is consistent with the in situ formation scenario supported by earlier works. For comparison, we estimate $R_{\text {standoff }}$ using the measured range of ionized gas velocities detected near P35 relative to the star's velocity from Dong et al. (2015). We find values for $R_{\text {standoff }}$ between $\sim 0.5$ and $0.1 \mathrm{pc}$, which are smaller than the observed size of $\mathrm{H} 2 \mathrm{NE}$, suggesting that the star is not an escapee.

In contrast to P35, the origin of the star P114 is less clear. The apparent cometary morphology of $\mathrm{H} 1$ might suggest that P114 is an interloper; however, Dong et al. (2015) show that the star has a comparable velocity to its surrounding ionized gas. This evidence may point to in situ formation, although these spectroscopic measurements could not completely distinguish between these scenarios. FORCAST observations of $\mathrm{H} 1$ reveal a nearly circular morphology for the $\mathrm{H}$ II region (Figure 9). This suggests that H1 may not be an escapee, given that the corresponding bow shock would produce a thin parabolic shell rather than a circular "bubble" around the source. A simple density gradient across the $\mathrm{H}$ II region could easily account for this shape, allowing for the star to be formed in situ.
To examine the origin of P114 further, we calculate $R_{\text {Strömgren }}$ for the H II region based on physical parameters of the star. Zhao et al. (1993) measured properties of H1, including the electron density $\left(n_{e}=5 \times 10^{2} \mathrm{~cm}^{-3}\right)$ and number of ionizing photons $\left(\log \left(N_{\mathrm{LyC}}\left[\mathrm{s}^{-1}\right]\right)=49.0\right)$. Based on these parameters, we find $R_{\text {Strömgren }}=1.1$ pc. While $\mathrm{H} 1$ is one of the largest H II regions in the complex (with a diameter of $0.9 \pm 0.08 \mathrm{pc}$, or alternatively, $\quad R_{\max }=0.75 \pm 0.08 \quad \mathrm{pc} \quad$ and $\quad R_{\min }=0.15 \pm$ $0.08 \mathrm{pc}$ measured from the location of P114), its radius is a factor of $\sim 2$ smaller than $R_{\text {Strömgren }}$. The smaller observed size could indicate that the $\mathrm{HII}$ region is still expanding to its equilibrium configuration, but we should also consider alternative explanations. We calculate the standoff radius for the $\mathrm{H}$ II region following the same procedure as $\mathrm{H} 2 \mathrm{NE}$ and find values between $\sim 0.07$ and $0.2 \mathrm{pc}$. These values are smaller than the average radius of the $\mathrm{H} 1$ "bubble" ( $\sim 0.45 \mathrm{pc})$; however, P114 is offset from the center of this structure. The minimum apparent distance between the star and the edge of the H II region is much smaller $\left(R_{\min }=0.15 \pm 0.08 \mathrm{pc}\right)$ and falls within the calculated range of $R_{\text {standoff }}$. Considering that both the calculated size and morphology of $\mathrm{H} 1$ support different origins for $\mathrm{P} 114$, it is difficult to state with certainty that this source is an escapee or formed in situ.

In addition to $\mathrm{H} 1$ and $\mathrm{H} 2$, we also consider the origins of stars associated with $\mathrm{H} 3$ and $\mathrm{H} 5$. The observed $19.7 \mu \mathrm{m}$ FWHM for each of these sources is $1.2 \pm 0.07 \mathrm{pc} \times$ $0.6 \pm 0.07 \mathrm{pc}$ for $\mathrm{H} 3,{ }^{18} 0.42 \pm 0.07 \mathrm{pc} \times 0.27 \pm 0.07 \mathrm{pc}$ for $\mathrm{H} 5 \mathrm{~W}$, and $0.39 \pm 0.07 \mathrm{pc} \times 0.39 \pm 0.07 \mathrm{pc}$ for $\mathrm{H} 5 \mathrm{E}$. While we have identified candidate stellar sources associated with each of these objects, relatively little is known about the stars. Fortunately, Zhao et al. (1993) provide measurements for $n_{e}$ and $N_{\mathrm{LyC}}$ for the $\mathrm{H}$ II regions. We calculate $R_{\text {Strömgren }}$ for

\footnotetext{
18 Here we consider the entirety of $\mathrm{H} 3$ rather than breaking it into a northern and southern component.
} 
each object based on these values and find $0.7 \mathrm{pc}$ for $\mathrm{H} 3,1.0 \mathrm{pc}$ for $\mathrm{H} 5 \mathrm{~W}$, and $0.8 \mathrm{pc}$ for $\mathrm{H} 5 \mathrm{E}$. For comparison, we also calculate $R_{\text {standoff }}$ for each object, assuming stellar velocities between 0 and $-20 \mathrm{~km} \mathrm{~s}^{-1}$, which are consistent with Arches cluster escapees from Habibi et al. (2014). This results in $R_{\text {standoff }}$ in the range of $\sim 0.6-1.8 \mathrm{pc}$ for $\mathrm{H} 3, \sim 0.7-2.0 \mathrm{pc}$ for $\mathrm{H} 5 \mathrm{~W}$, and $\sim 0.7-2.0 \mathrm{pc}$ for H5E. Because the span of $R_{\text {standoff }}$ values overlaps with the calculated $R_{\text {Strömgren }}$ values, we cannot differentiate between these scenarios using this method. However, it is worth noting that the observed and calculated sizes of the H II regions do not agree in either case. Perhaps this is due to irregularities with these $\mathrm{H} \mathrm{II}$ regions that violate assumptions made in the calculations. For example, the elongated shape of $\mathrm{H} 3$ may be due to multiple sources within the region, which we previously inferred from the dust-heating analysis. Additionally, P107 may be external to H5, which could present a very different geometry than what is assumed. However, the inference of an external ionization source would certainly imply that the star was not formed in situ.

While much of our discussion has focused on the spatially extended H II regions, the origin of the more compact and nondetected sources is also worth examining. The observed infrared emission of these objects can be used as an indicator of dust in the stars' local surroundings, which would be expected for the natal environment of a young star. For example, H2NE is consistent with being formed in situ and is a luminous midinfrared source with a relatively high dust temperature. In contrast, the sources $\mathrm{H} 9$ and $\mathrm{H} 13$ are not detected in the FORCAST maps even though they are strong radio continuum and $\mathrm{Pa} \alpha$ sources. This suggests that the stars associated with these $\mathrm{H}$ II regions have little dust in their immediate surroundings and are likely cluster escapees. Other H II regions in the complex with relatively weak mid-infrared emission are difficult to assess with this line of reasoning because their associated stellar sources are not well characterized. ${ }^{19}$ Differences in the infrared luminosity of these sources compared to the more luminous objects like $\mathrm{H} 2$ could simply be indicative of a lower-luminosity star rather than an absence of nearby dust. The only other source that shows evidence of being an escapee is H10, whose near-infrared morphology suggests that it is likely an interloper (Figure 8).

Overall, our study of the $\mathrm{H} \mathrm{H}$ II regions suggests that they do not have a uniform origin. Based on their kinematics and morphology, we favor P35 forming in situ. However, the stars associated with $\mathrm{H} 9,10$, and $\mathrm{H} 13$ are better explained as cluster escapees. The origin of $\mathrm{H} 1$ and the remaining sources in the complex are ambiguous, and further study is needed to better understand these objects.

\subsection{Small Clusters in the H II Regions?}

Recently, Dong et al. (2017) examined an apparent overdensity of stars near the location of P35 that may be associated with $\mathrm{H} 2$. A number of these stars have similar near-infrared color to P35, which suggests that they are indeed associated and may be members of a stellar cluster. If true, this result is significant because little is known about clusters in the GC outside of the Arches, Quintuplet, and Central clusters. If $\mathrm{H} 2$ is P35's natal environment, it is reasonable to expect there to be other members of a cluster that have formed nearby. In principle, these stars should contribute to the observed

\footnotetext{
19 With the exception of H14.
}

luminosity of the $\mathrm{HII}$ region, and their presence may be inferred via excess infrared luminosity. Similarly, we can search for luminous stellar clusters associated with the other $\mathrm{H}$ II regions in this complex.

The SED models of $\mathrm{H} 1, \mathrm{H} 2, \mathrm{H} 3$, and $\mathrm{H} 5$ have infrared luminosities ranging from $\sim 3 \times 10^{5}$ to $6 \times 10^{5} L_{\odot}$, which are consistent with individual massive stars. However, we cannot easily exclude the presence of a relatively small cluster (with total luminosity $\sim 10^{5} L_{\odot}$ ). Such a cluster could reasonably exist, given that the total luminosity is dominated by the hottest (in this case O-type) stars. ${ }^{20}$ If any of the $\mathrm{H}$ II regions have a high escape fraction of photons (similar to H14), it may be possible to hide an even more luminous cluster $\left(\sim 10^{6}-10^{7} L_{\odot}\right)$; however, a small escape fraction would not allow for such a scenario. Our measurements cannot distinguish between these possibilities, but the prospect of stellar clusters associated with these $\mathrm{H}$ II regions merits further study.

\subsection{Additional Infrared Sources}

The infrared sources $\mathrm{H} 15-\mathrm{H} 23 \mathrm{c}$ (Figure 1) are very interesting because they could be compact HII regions or another type of luminous dust-enshrouded star. Differentiating between these scenarios is difficult with the data currently in hand, though we are planning a more complete analysis of these sources for a future paper. In this work, we provide locations of the sources and an estimate of their infrared luminosity, determined by integrating over the observed source photometry from 3.6 to $37.1 \mu \mathrm{m}$. Because of heavy backgrounds in the region at far-infrared wavelengths, it is unclear whether any of the sources have a $70 \mu \mathrm{m}$ counterpart. We calculate the color temperatures for the few sources that are detected at both 19.7 and $37.1 \mu \mathrm{m}$. We also provide likely counterparts for the identified FORCAST sources in the IRAC GC catalog (Ramírez et al. 2008). A summary of these sources can be found in Table 5. Of the nine sources we identify, five were included in the earlier study by An et al. (2011) of infrared sources in the GC. All five were determined not to be massive YSOs, but further work is needed to classify each source and the remaining four not included in the An et al. (2011) sample.

\section{Conclusions}

In this work, we examine the $\mathrm{H} \mathrm{H}$ II regions in the $\mathrm{GC}$ using observations from SOFIA/FORCAST and other data from the literature. We create color-temperature maps of $\mathrm{H} 1, \mathrm{H} 2, \mathrm{H} 3$, and $\mathrm{H} 5$ to analyze their heating structure. We show that $\mathrm{H} 1$ and H2NE are likely heated by their candidate ionizing sources, $\mathrm{P} 114$ and P35. This analysis also indicates that P107 is not the primary heating source of H5. We identify candidate heating sources for the infrared components of the H II regions, including $\mathrm{H} 2 \mathrm{SW}, \mathrm{H} 3 \mathrm{~S}, \mathrm{H} 3 \mathrm{~N}, \mathrm{H} 5 \mathrm{E}$, and H5W.

In addition to our study of dust temperatures, we also fit SEDs for each of the H II regions to constrain their physical properties. We show that most of the objects appear depleted in PAHs, while $\mathrm{H} 4$ and $\mathrm{H} 11$ are consistent with a typical ISM PAH abundance and $\mathrm{H} 14$ appears to have an enhanced abundance of PAHs. The infrared luminosity of the H II

\footnotetext{
${ }^{20}$ One can easily show that for a population of stars containing a single late O-type star and following a Salpeter initial mass function (Salpeter 1955), the corresponding B-type stars have a combined luminosity that is similar to the O-type star.
} 
Table 5

Newly Identified Infrared Sources

\begin{tabular}{|c|c|c|c|c|c|c|c|c|}
\hline Source ID & Location $(l, b)^{\mathrm{a}}$ & $F_{19.7}(\mathrm{Jy})$ & $F_{25.2}(\mathrm{Jy})$ & $F_{31.5}(\mathrm{Jy})$ & $F_{37.1}(\mathrm{Jy})$ & $T_{D}(\mathrm{~K})$ & $L_{\mathrm{IR}}\left(L_{\odot}\right)$ & IRAC Counterpart $^{\mathrm{b}}$ \\
\hline H15 & $(00.054,00.034)$ & $10.9 \pm 4.9$ & $18.0 \pm 7.8$ & $18.7 \pm 7.1$ & $22.9 \pm 7.7$ & 120 & $5.2 \times 10^{3}$ & SSTGC $516435^{\mathrm{c}}$ \\
\hline H16 & $(00.033,00.019)$ & $72.5 \pm 22.2$ & $71.0 \pm 20.0$ & $\ldots^{d}$ & $29.1 \pm 8.9$ & 310 & $2.6 \times 10^{4}$ & SSTGC $517724^{\mathrm{c}}$ \\
\hline H17 & $(00.028,00.016)$ & $11.4 \pm 6.3$ & $11.0 \pm 5.7$ & $5.67 \pm 3.35$ & $\ldots$ & $\cdots$ & $3.8 \times 10^{3}$ & SSTGC 517943 \\
\hline H18 & $(00.015,00.025)$ & $5.37 \pm 4.60$ & $10.5 \pm 5.6$ & $9.04 \pm 4.50$ & $11.8 \pm 4.9$ & 120 & $2.8 \times 10^{3}$ & SSTGC $507261^{\mathrm{c}}$ \\
\hline H19 & $(359.999,00.029)$ & $31.3 \pm 12.4$ & $40.2 \pm 13.0$ & $31.1 \pm 9.8$ & $29.6 \pm 9.0$ & 160 & $1.2 \times 10^{4}$ & SSTGC 498604 \\
\hline $\mathrm{H} 20$ & $(00.003,00.016)$ & $11.8 \pm 6.9$ & $17.8 \pm 7.7$ & $12.1 \pm 5.4$ & $10.9 \pm 4.7$ & 160 & $4.9 \times 10^{3}$ & SSTGC 508015 \\
\hline $\mathrm{H} 21$ & $(359.990,00.030)$ & $4.16 \pm 3.87$ & $3.61 \pm 3.10$ & $\ldots$ & $\ldots$ & $\ldots$ & $4.6 \times 10^{3}$ & SSTGC 0494496 \\
\hline $\mathrm{H} 22 \mathrm{c}$ & $(00.039,00.013)$ & $7.18 \pm 5.00$ & $\ldots$ & $\ldots$ & $\cdots$ & $\cdots$ & $1.7 \times 10^{3}$ & SSTGC $524419^{c}$ \\
\hline $\mathrm{H} 23 \mathrm{c}$ & $(00.022,00.015)$ & $5.67 \pm 4.2$ & $\cdots$ & $\cdots$ & $\cdots$ & $\cdots$ & $1.3 \times 10^{3}$ & SSTGC $516756^{\mathrm{c}}$ \\
\hline
\end{tabular}

Notes.

${ }^{\mathrm{a}}$ The location of the observed $19.7 \mu \mathrm{m}$ source Galactic coordinates. Typical errors in the position are $\lesssim 1$ !' 5 .

b IRAC photometry of these sources was taken from the Spitzer/IRAC survey of the GC point-source catalog (Ramírez et al. 2008).

c These IRAC sources have been ruled out as massive YSOs in an earlier spectroscopic study by An et al. (2011).

d At $31.5 \mu \mathrm{m}$ the location of H16 fell on the edge of two FORCAST fields and its flux could not be reliably recovered.

regions ranges from $L_{\mathrm{IR}} \approx 3 \times 10^{3}$ to $6 \times 10^{5} L_{\odot}$. The sources $\mathrm{H} 4, \mathrm{H} 6, \mathrm{H} 7, \mathrm{H} 10, \mathrm{H} 11$, and $\mathrm{H} 14$ have luminosities that are characteristically smaller than $\mathrm{H} 1, \mathrm{H} 2, \mathrm{H} 3$, and $\mathrm{H} 5$. These variations may indicate differences in the intrinsic luminosity of the heating sources or differences in the amount of radiation that is absorbed by dust and re-emitted in the infrared. It is possible that one or more of these sources could contain a stellar cluster as suggested by Dong et al. (2017); however, it is difficult to test this hypothesis with our data because the infrared luminosity of the $\mathrm{HII}$ regions only provides a lower limit on the total luminosity of the sources.

We also examine possible origins of massive stars associated with the $\mathrm{H}$ II regions. The calculated Strömgren radius of $\mathrm{H} 2$ indicates that $\mathrm{H} 2 \mathrm{NE}$ is consistent with an in situ formation scenario for P35. In contrast, the weak infrared emission associated with H9 and H13 implies that these sources are likely associated with interlopers. The near-infrared morphology of $\mathrm{H} 1$ and $\mathrm{H} 10$ suggests a similar origin for these objects; however, the morphology of sources can be challenging to interpret, as there is evidence that $\mathrm{H} 1$ is not a simple bow shock. The complex structure of these and other sources in the complex makes their interpretation difficult and could explain discrepancies with the calculated $\mathrm{H}$ II region sizes as in the case of $\mathrm{H} 3$ and H5. In any case, the inhomogeneous origin of sources in this complex highlights the need for careful study of other GC field stars.

Ultimately, the FORCAST data have helped to reveal a number of interesting features associated with the $\mathrm{H} \mathrm{H}$ II regions, including several newly identified infrared sources that may be part of this complex. Future studies of these sources would benefit from more complete near-infrared data for stars in the region, although our constraints on the heating sources and suggested candidates should prove useful. Our observations also show the utility of FORCAST for finding dusty, luminous, nonionizing sources in the GC (similar to H2SW). In the future, a survey for these types of sources in the GC could significantly bolster our understanding of the distribution of luminous infrared sources and provide clues on the origin of additional massive field stars in the region.

We thank the rest of the FORCAST team, including George Gull, Justin Schoenwald, Chuck Henderson, and Joe Adams; the USRA Science and Mission Ops teams; and the entire
SOFIA staff. We also thank the reviewer for their insightful comments, which helped improve this manuscript. This work is based on observations made with the NASA/DLR Stratospheric Observatory for Infrared Astronomy (SOFIA). SOFIA science mission operations are conducted jointly by the Universities Space Research Association, Inc. (USRA), under NASA contract NAS2-97001, and the Deutsches SOFIA Institut (DSI) under DLR contract 50 OK 0901. Financial support for FORCAST was provided by NASA through award 8500-98-014 issued by USRA. This research has made use of the VizieR catalog access tool, CDS, Strasbourg, France. The original description of the VizieR service was published in A\&AS 143, 23. This publication also makes use of data products from the Two Micron All Sky Survey, which is a joint project of the University of Massachusetts and the Infrared Processing and Analysis Center/California Institute of Technology, funded by the National Aeronautics and Space Administration and the National Science Foundation. This material is based on work supported by the National Science Foundation Graduate Research Fellowship under grant No. DGE-1144153.

\section{ORCID iDs}

M. J. Hankins (1D https://orcid.org/0000-0001-9315-8437 E. A. C. Mills (D) https://orcid.org/0000-0001-8782-1992 M. R. Morris (ib https://orcid.org/0000-0002-6753-2066 T. L. Herter (ib https://orcid.org/0000-0002-3856-8385

\section{References}

An, D., Ramírez, S. V., Sellgren, K., et al. 2011, ApJ, 736, 133 Barnes, A. T., Longmore, S. N., Battersby, C., et al. 2017, MNRAS, 469, 2263 Cardelli, J. A., Clayton, G. C., \& Mathis, J. S. 1989, ApJ, 345, 245 Churchwell, E., Babler, B. L., Meade, M. R., et al. 2009, PASP, 121, 213 Compiègne, M., Verstraete, L., Jones, A., et al. 2011, A\&A, 525, A103 Cotera, A. S., Erickson, E. F., Colgan, S. W. J., et al. 1996, ApJ, 461, 750 Cutri, R. M., Skrutskie, M. F., van Dyk, S., et al. 2003, 2MASS All Sky Catalog of Point Sources (Pasadena: IPAC/Caltech)

de Pree, C. G., Gaume, R. A., Goss, W. M., \& Claussen, M. J. 1996, ApJ, 464, 788

De Pree, C. G., Goss, W. M., \& Gaume, R. A. 1998, ApJ, 500, 847

Dong, H., Lacy, J. H., Schödel, R., et al. 2017, MNRAS, 470, 561

Dong, H., Mauerhan, J., Morris, M. R., Wang, Q. D., \& Cotera, A. 2015, MNRAS, 446, 842

Dong, H., Wang, Q. D., Cotera, A., et al. 2011, MNRAS, 417, 114 
Draine, B. T. 2011, Physics of the Interstellar and Intergalactic Medium (Princeton, NJ: Princeton Univ. Press)

Egan, M., Price, S., Kraemer, K., et al. 2003, Air Force Research Laboratory Technical Report AFRL-VS-TR-2003-1589, ADA418993

Ekers, R. D., van Gorkom, J. H., Schwarz, U. J., \& Goss, W. M. 1983, A\&A, 122,143

Figer, D. F. 2004, in ASP Conf. Ser. 322, The Formation and Evolution of Massive Young Star Clusters, ed. H. J. G. L. M. Lamers, L. J. Smith, \& A. Nota (San Francisco, CA: ASP), 49

Figer, D. F., Kim, S. S., Morris, M., et al. 1999, ApJ, 525, 750

Fritz, T. K., Gillessen, S., Dodds-Eden, K., et al. 2011, ApJ, 737, 73

Gaume, R. A., Claussen, M. J., de Pree, C. G., Goss, W. M., \& Mehringer, D. M. 1995, ApJ, 449, 663

Goldsmith, P. F., Lis, D. C., Hills, R., \& Lasenby, J. 1990, ApJ, 350, 186

Habibi, M., Stolte, A., \& Harfst, S. 2014, A\&A, 566, A6

Hankins, M. J., Lau, R. M., Morris, M. R., \& Herter, T. L. 2017, ApJ, 837, 79

Herter, T. L., Adams, J. D., De Buizer, J. M., et al. 2012, ApJL, 749, L18

Herter, T. L., Vacca, W. D., Adams, J. D., et al. 2013, PASP, 125, 1393

Hinz, J., Rieke, G., Yusef-Zadeh, F., et al. 2009, ApJS, 181, 227

Kruijssen, J. M. D., Longmore, S. N., Elmegreen, B. G., et al. 2014, MNRAS, 440, 3370

Lamers, H. J. G. L. M., \& Leitherer, C. 1993, ApJ, 412, 771

Lang, C. C., Goss, W. M., \& Morris, M. 2001, AJ, 121, 2681

Lau, R. M., Hankins, M. J., Herter, T. L., et al. 2016, ApJ, 818, 117

Lau, R. M., Herter, T. L., Morris, M. R., \& Adams, J. D. 2014, ApJ, 794, 108

Longmore, S. N., Bally, J., Testi, L., et al. 2013, MNRAS, 429, 987

Lucy, L. B. 1974, AJ, 79, 745

Martins, F., Schaerer, D., \& Hillier, D. J. 2005, A\&A, 436, 1049
Mathis, J. S., Mezger, P. G., \& Panagia, N. 1983, A\&A, 128, 212

Mauerhan, J. C., Cotera, A., Dong, H., et al. 2010, ApJ, 725, 188

McKee, C. F., \& Ostriker, E. C. 2007, ARA\&A, 45, 565

Mills, E., Morris, M. R., Lang, C. C., et al. 2011, ApJ, 735, 84

Molinari, S., Bally, J., Noriega-Crespo, A., et al. 2011, ApJL, 735, L35

Molinari, S., Swinyard, B., Bally, J., et al. 2010, PASP, 122, 314

Nagata, T., Woodward, C. E., Shure, M., Pipher, J. L., \& Okuda, H. 1990, ApJ, 351,83

Nogueras-Lara, F., Gallego-Calvente, A. T., Dong, H., et al. 2018, A\&A, 610, A83

Okuda, H., Shibai, H., Nakagawa, T., et al. 1990, ApJ, 351, 89

Pecaut, M. J., \& Mamajek, E. E. 2013, ApJS, 208, 9

Poglitsch, A., Stacey, G. J., Geis, N., et al. 1991, ApJL, 374, L33

Ramírez, S. V., Arendt, R. G., Sellgren, K., et al. 2008, ApJS, 175, 147

Richardson, W. H. 1972, JOSA, 62, 55

Salgado, F., Berné, O., Adams, J. D., et al. 2016, ApJ, 830, 118

Salpeter, E. E. 1955, ApJ, 121, 161

Serabyn, E., \& Guesten, R. 1987, A\&A, 184, 133

Steinke, M., Oskinova, L. M., Hamann, W.-R., et al. 2016, A\&A, 588, A9

Strömgren, B. 1939, ApJ, 89, 526

Tielens, A. G. G. M., \& Hollenbach, D. 1985, ApJ, 291, 722

Wang, Q. D., Dong, H., Cotera, A., et al. 2010, MNRAS, 402, 895

Wilkin, F. P. 1996, ApJL, 459, L31

Young, E. T., Becklin, E. E., Marcum, P. M., et al. 2012, ApJL, 749, L17

Yusef-Zadeh, F., Hewitt, J. W., \& Cotton, W. 2004, ApJS, 155, 421

Yusef-Zadeh, F., Lacy, J. H., Wardle, M., et al. 2010, ApJ, 725, 1429

Yusef-Zadeh, F., \& Morris, M. 1987, ApJ, 320, 545

Zhao, J.-H., Desai, K., Goss, W. M., \& Yusef-Zadeh, F. 1993, ApJ, 418, 235 